\title{
Blind Interference Alignment for Cellular Networks
}

\author{
Máximo Morales-Céspedes, Student Member, IEEE, Jorge Plata-Chaves, Member, IEEE, Dimitris \\ Toumpakaris, Member, IEEE, Syed A. Jafar, Fellow Member, IEEE, and Ana García Armada, Senior \\ Member, IEEE
}

\begin{abstract}
We propose a blind interference alignment scheme for partially connected cellular networks. The scheme cancels both intracell and intercell interference by relying on receivers with one reconfigurable antenna and by allowing users at the cell edge to be served by all the base stations in their proximity. An outer bound for the Degrees of Freedom is derived for general partially connected networks with single-antenna receivers when knowledge of the channel state information at the transmitter is not available. It is demonstrated that for symmetric scenarios this outer bound is achieved by the proposed scheme. On the other hand, for asymmetric scenarios the achievable Degrees of Freedom are not always equal to the outer bound. However, the penalty is typically small, and the proposed scheme outperforms other blind interference alignment schemes. Moreover, significant reduction of the supersymbol length is achieved compared to a standard blind interference alignment strategy designed for fully connected networks.
\end{abstract}

Index Terms-Blind Interference Alignment, Cellular Networks, Degrees of Freedom.

\section{INTRODUCTION}

$\mathbf{T}$ HE pressing need to improve the efficiency of wireless systems has led to the intensive study of interference and its effect on communication. Until fairly recently, the typical design approach was to avoid interference as much as possible. Lately, there has been a gradual shift to operating in the presence of interference. Interference Alignment (IA) is based on this approach [1]. The aim of IA is to ensure that, at each receiver, all interference is contained in a signal subspace with the smallest possible dimension. It is then possible to

Máximo Morales Céspedes and Ana García Armada are with the Department of Signal Theory and Communications, Universidad Carlos III de Madrid, Avda. Universidad 30, 28911, Leganés (Madrid), Spain (email: maximo@tsc.uc3m.es, agarcia@tsc.uc3m.es). This work has been partially funded by research projects COMONSENS (CSD2008-00010) and GRE3N(TEC2011-29006-C03-02).

Jorge Plata-Chaves is with the Department of Electrical Engineering (ESATSCD/ SISTA), Katholieke Universiteit Leuven, B-3001 Leuven, Belgium (email: jplata@esat.kuleuven.be). This research work was partly carried out at the ESAT Laboratory of KU Leuven in the frame of the Belgian Programme on Interuniversity Attractive Poles Programme initiated by the Belgian Science Policy Office: IUAP P7/23 'Belgian network on stochastic modeling analysis design and optimization of communication systems' (BESTCOM) 2012-2017.

Dimitris Toumpakaris is with the Department of Electrical \& Computer Engineering, University of Patras, 26500, Rio Achaias, Greece (email: dtouba@upatras.gr). The work of D. Toumpakaris was supported by the European Union (European Social Fund - ESF) and Greek national funds through the Operational Program Education and Lifelong Learning of the National Strategic Reference Framework through the Research Funding Program Thales - Investing in knowledge society through the European Social Fund.

Syed A. Jafar is with the Department of Electrical Engineering and Computer Sciences, University of California, Irvine, CA, 92697 USA (e-mail: syed@uci.edu). The work of Syed Jafar was supported in part by NSF grants CCF-1319104 and CCF-1317351.

Copyright (c) 2014 IEEE. Personal use of this material is permitted. However, permission to use this material for any other purposes must be obtained from the IEEE by sending a request to pubs-permissions@ieee.org. cancel the effect of interference by projecting the received signal onto the orthogonal subspace of the subspace containing the interference [2].

The concept of Degrees of Freedom (DoF) is often employed to characterize the performance of variants of IA in the high SNR regime [3], [4]. It has been shown that, for several scenarios, IA attains the optimal DoF. Several variants of IA exist, depending on the amount of channel knowledge that is available at the transmitter, the scenario over which IA is applied, and the channel statistics. An overview of IA is given in [5].

An important assumption of the first IA schemes that were proposed was perfect Channel State Information is available at the Transmitter (CSIT). This requirement is often challenging or even impossible to satisfy in a realistic implementation [6]. Recently, a technique called Blind Interference Alignment (BIA) was proposed for the Multiuser Multiple-Input Single-Output (MU-MISO) Broadcast Channel that achieves a growth in DoF compared with orthogonal techniques such as TDMA or FDMA [2], [7], [8]. As demonstrated in [7], if the transmitter is equipped with $N_{t}$ antennas that serve $K_{t o t}$ single-antenna users, the sum DoF that is achieved by BIA is $\frac{N_{t} K_{t o t}}{N_{t}+K_{t o t}-1}$, which is also the outer bound for this setting [9].

As will be discussed in more detail in Section II, the BIA scheme of [7] requires that the channel not change during one supersymbol. Therefore, coherence time or bandwidth is important when determining whether BIA can be used. This motivates the search for BIA schemes that require short supersymbols. Moreover, each user needs to be equipped with a reconfigurable antenna whose function is to switch its radiation pattern among a set of preset modes [10]. Although this adds complexity to the receiver, there has been active interest and recent progress in the area, which makes it likely that such receivers may be affordable in the future.

The BIA scheme of [7], which will be called standard BIA (sBIA) from now on, was devised for one multiple-antenna base station (BS). Clearly, it is of interest to investigate how the scheme can be applied to cellular systems and what the achievable rates are. The performance of sBIA in cellular and cluster systems was analyzed in [11]. It was shown that the rates of the users located at the cell edge can be poor because of intercell interference. In [12], ways to apply sBIA to cellular scenarios such as Frequency Reuse (FR) were proposed and were compared to Linear Zero Forcing Beamforming (LZFB) [13] taking into account the cost of CSIT. One interesting observation in [11] and [12] was that, if the BIA codes of the BSs of neighboring cells are synchronized, intercell interference can be reduced considerably. In addition to coordination among the BSs, the authors in [14] derive a scheme that relies 
on data sharing when transmitting data to cell-edge users. This way, intercell interference is completely cancelled. Although this scheme improves significantly the rates of cell-edge users at low SNRs, there is a loss in DoF because of the identical data that are sent by all BSs that transmit to cell-edge users.

In order to maximize the achievable DoF over the entire cellular system when using BIA, a straightforward approach would be to apply a fully coordinated BIA scheme (cBIA) among all BSs in the system. Assuming $N_{B S}$ BSs each with $N_{t}$ antennas, the total number of antennas is equal to $M=N_{B S} \cdot N_{t}$. If $K_{t o t}$ is the number of active users in the entire network, $\frac{M K_{t o t}}{M+K_{t o t}-1}$ DoF could potentially be achieved. Clearly, such an approach would be costly in practice because of the need for all BSs to share data of all users. Moreover, because of the large number of antennas and users, the length of the supersymbol could be large, meaning that large channel coherence time or bandwidth would also be necessary. Last but not least, full connectivity would be required in the system, which is generally not true in several practical scenarios. Due to the partial connectivity [15], only signals of a small number of BSs can be decoded at each user. Users at the cell edge can receive data with an acceptable Signal-to-Noise ratio. In contrast, for users located near a BS, the signals from other BSs are weaker and their decoding is strongly handicapped by the noise power.

At first sight, it may appear that partial connectivity leads to a loss in DoF. Interestingly, this is not the case. A major objective of this paper is to demonstrate that, owing to the partial connectivity, use of BIA can actually lead to more DoF than if the system were fully connected. In retrospect, this is not surprising. The same way that large path loss can help increase spectral efficiency by allowing frequency reuse, partial connectivity allows simultaneous transmission of more data streams compared to a fully connected network. As an example, in [16] it is shown that, in a $K$-user interference channel, there exist scenarios where treating interference as noise achieves all points in the capacity region up to a constant gap, namely it is DoF-optimal.

In this paper, a network BIA (nBIA) scheme is proposed for partially connected cellular networks. The scheme differentiates between "private" users near the BSs who treat intercell interference as noise and "shared" users at the cell edge who are connected to all BSs in their proximity. Unlike [14], the BSs do not share data. Instead, each BS handles the transmission of part of the overall data stream. For the symmetric scenario, where the number of private users, $K_{p}$, in each cell is the same, it is shown that the proposed scheme is DoF-optimal. Moreover, as will be shown, owing to the partially connected topology, fewer reconfigurable modes are needed for the private users. Finally, the nBIA supersymbol is shorter than cBIA. This relaxes the requirements for the coherence time or bandwidth, and renders the scheme attractive for practical implementation.

The remainder of this paper is organized as follows. In Section II the system model is presented. Section III introduces a toy example to provide an overview of cBIA and, at the same time, motivates our work. Section IV presents the network BIA (nBIA) scheme for a symmetric cellular network with

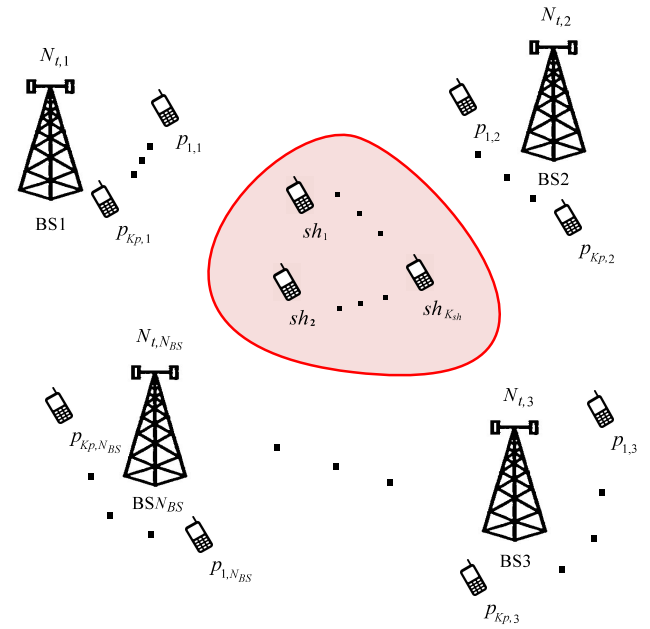

Fig. 1. Cellular system with partial connectivity and $N_{B S}$ BSs. Each BS is equipped with $N_{t, n}$ antennas and serves $K_{p, n}$ private users as well as $K_{s h}$ shared users together with the other BSs.

partial connectivity. In Section $\mathrm{V}$, we provide an outer bound for the sum-DoF in a partially connected network. From this outer bound, we show that nBIA is DoF-optimal for symmetric scenarios. An extension of the nBIA scheme for asymmetric user distributions is presented in Section VI. In Section VII closed-form expressions are derived for the rates achieved by nBIA. Section VIII shows several simulation results where the performance of nBIA is compared to other BIA schemes. Finally, Section IX provides concluding remarks.

\section{System MODEL}

We consider a set of $N_{B S}$ Base Stations (BSs) $\mathcal{N}=$ $\left\{1,2, \ldots, N_{B S}\right\}$ that want to send a set of messages to $K_{t o t}$ users in a partially connected cellular network as shown in Fig. 1. Each $\mathrm{BS} n, n \in \mathcal{N}$, has $N_{t, n}$ transmit antennas and wishes to send data to a set of private users $\mathcal{K}_{p, n}=\left\{p_{1, n}, \ldots, p_{K_{p, n}, n}\right\}$ as well as a set of shared users $\mathcal{K}_{s h}=\left\{s h_{1}, \ldots, s h_{K_{s h}}\right\}$ located on the edge of all $N_{B S}$ cells.

Each private user is equipped with one reconfigurable antenna that can switch among $N_{t, n}$ preset modes, whereas each shared user can switch among $M=\sum_{n=1}^{N_{B S}} N_{t, n}$ modes ${ }^{1}$. Therefore, if $m^{\left[p_{k, n}\right]}[i]$ denotes the antenna mode of private user $p_{k, n}$ of $\mathrm{BS} n$ at time $i$, the signal received by the user at time $i$ can be written as

$$
y^{\left[p_{k, n}\right]}[i]=\mathbf{h}^{\left[p_{k, n}\right]}\left(m^{\left[p_{k, n}\right]}[i]\right)^{T} \mathbf{x}[i]+z^{\left[p_{k, n}\right]}[i],
$$

where $z^{\left[p_{k, n}\right]}[i] \sim \mathcal{C N}(0,1)$ is additive white Gaussian noise $(\mathrm{AWGN})$,

$$
\mathbf{x}[i]=\left[\mathbf{x}^{[1]}[i]^{T} \quad \mathbf{x}^{[2]}[i]^{T} \quad \cdots \quad \mathbf{x}^{\left[N_{B S}\right]}[i]^{T}\right]^{T} \in \mathbb{C}^{M \times 1},
$$

\footnotetext{
${ }^{1}$ In practice, in a network with user mobility, each user should be able to switch among $M$ preset modes, since it may transition from being private to being shared and vice versa.
} 
and

$$
\begin{aligned}
\mathbf{h}^{\left[p_{k, n}\right]} & =\left[\begin{array}{lll}
\mathbf{h}^{\left[p_{k, n}, 1\right]^{T}} \ldots & \mathbf{h}^{\left[p_{k, n}, n\right]^{T}} \ldots & \left.\mathbf{h}^{\left[p_{k, n}, N_{B S}\right]}\right]^{T}
\end{array}\right]^{T} \\
& \approx\left[\begin{array}{lll}
\mathbf{0}_{a, 1}{ }^{T} & \mathbf{h}^{\left[p_{k, n}, n\right]^{T}} & \mathbf{0}_{b, 1}{ }^{T}
\end{array}\right]^{T} \in \mathbb{C}^{M \times 1}
\end{aligned}
$$

with $a=\sum_{n^{\prime}=1}^{n-1} N_{t, n^{\prime}}, b=\sum_{n^{\prime}=n+1}^{N_{B S}} N_{t, n^{\prime}}$ and $\mathbf{0}_{c, 1}$ is a vector of zeros of dimension $c \times 1$. In (2), $\mathbf{x}^{[n]}[i] \in \mathbb{C}^{N_{t, n} \times 1}$ is the signal sent by BS $n$ at time $i$, whereas in (3), $\mathbf{h}^{\left[p_{k, n}, n\right]}(m)=$ $\left[h_{1}^{\left[p_{k, n}, n\right]}(m)^{T} \quad \cdots \quad h_{N_{t}, n}^{\left[p_{k, n}, n\right]}(m)^{T}\right]^{T} \in \mathbb{C}^{N_{t, n} \times 1}$ contains the channel coefficients between the $N_{t, n}$ antennas of BS $n$ and the single antenna of private user $p_{k, n}$ when its radiation pattern is set to mode $m \in\left\{1,2, \ldots, N_{t, n}\right\}$. As can be seen in (3), we model the situation where the $K_{p, n}=\left|\mathcal{K}_{p, n}\right|$ private users of cell $n$ are close to BS $n$, and assume that signals received from any other BS $n^{\prime} \neq n$ are negligible. Thus, no data sharing among the BSs is required to serve the private users, and $\mathbf{x}^{\left[n^{\prime}\right]}[i]$ does not contain data intended to any private user $p_{k, n} \in \mathcal{K}_{p, n}, n \neq n^{\prime}$.

Similar to the model for the private users, the signal received by shared user $s h_{k^{\prime}}$ at time $i$ can be written as

$$
y^{\left[s h_{k^{\prime}}\right]}[i]=\mathbf{h}^{\left[s h_{k^{\prime}}\right]}\left(m^{\left[s h_{k^{\prime}}\right]}[i]\right)^{T} \mathbf{x}[i]+z^{\left[s h_{k^{\prime}}\right]}[i],
$$

where, $\mathbf{x}[i]$ is as defined in (2) and

$$
\mathbf{h}^{\left[s h_{k^{\prime}}\right]}=\left[\begin{array}{lll}
\mathbf{h}^{\left[s h_{k^{\prime}}, 1\right]^{T}} & \cdots & \left.\mathbf{h}^{\left[s h_{k^{\prime}}, N_{B S}\right.}\right]^{T}
\end{array}\right]^{T} \in \mathbb{C}^{M \times 1},
$$

with $M=\sum_{n^{\prime}=1}^{N_{B S}} N_{t, n}$ and $\mathbf{h}^{\left[s h_{k^{\prime}}, n\right]}(m) \in \mathbb{C}^{N_{t, n} \times 1}$ denoting the channel between the $N_{t, n}$ antennas of BS $n$ and shared user $s h_{k^{\prime}}$ for mode $m$. We use index $k^{\prime}$ instead of $k$ to distinguish from private users. It is assumed that shared users can receive signals from all BSs because of their location in the network. As a result, the task of sending data to the shared users can be jointly undertaken by the $N_{B S}$ BSs.

We also assume that the channel input is subject to an average power constraint $E\left\{\left\|\mathbf{x}^{[n]}[i]\right\|^{2}\right\} \leq P$ for all $i \geq 1$ and $n \in \mathcal{N}$. Furthermore, the channels between each user, whether private or shared, and the BSs are considered to be drawn from a continuous distribution and, therefore, are linearly independent almost surely. We also assume that the switching pattern functions $m^{\left[p_{k, n}\right]}[i]$ and $m^{\left[s h_{k^{\prime}}\right]}[i]$ are predetermined and are known to everyone in the system. On the contrary, we assume that the transmitters do not have any channel state information (CSIT). Moreover, we assume that the physical channels stay constant across a sufficient number of time or frequency slots. For simplicity, we focus on the temporal dimension, without loss of generality. Hence, from now on each symbol extension $i$ corresponds to a time slot. The application of the scheme along frequency slots is straightforward.

\section{Fully COOPERATIVE BLIND INTERFERENCE ALIGNMENT}

\section{A. A fully cooperative scheme}

The sBIA scheme can be extended to a cellular system in a straightforward way by creating a fully cooperative BIA (cBIA) scheme where, as in a network MIMO system, full connectivity and full data sharing among all BSs is assumed.
If $M=\sum_{n=1}^{N_{B S}} N_{t, n}$ antennas transmit to all $K_{t o t}$ users, which can switch among $M$ reconfigurable modes, following the scheme in [7] a supersymbol that contains $(M-1)^{K_{t o t}-1}$ alignment blocks per user, each providing $M$ DoF to the user, can be built over $(M-1)^{K_{t o t}}+K_{t o t}(M-1)^{K_{t o t}-1}$ symbol extensions. A generic cBIA supersymbol is shown in Fig. 2.

In the supersymbol, user $k$ switches among all $M$ preset modes during each alignment block, while the channels $\mathbf{h}^{\left[k^{\prime}\right]}(m)$ of all other users, $k^{\prime} \neq k$, remain in a specific preset mode. For example, in Fig. 2 the first alignment block of user 1 is composed by the first $(M-1)$ symbol extensions of Block 1 plus symbol extension $(M-1)^{K_{t o t}}+1$ (the first symbol extension of Block 2). Therefore, if we ignore the noise, a typical signal vector $\mathbf{Y}^{[k]}=\left[\begin{array}{llll}y^{[k]}[1] & \ldots & y^{[k]}[M]\end{array}\right]^{T}$ received by user $k$ in a given alignment block is given by

$$
\mathbf{Y}^{[k]}=\underbrace{\left[\begin{array}{c}
\mathbf{h}^{[k]}(1)^{T} \\
\vdots \\
\mathbf{h}^{[k]}(M-1)^{T} \\
\mathbf{h}^{[k]}(M)^{T}
\end{array}\right]}_{\mathbf{H}^{[k]}} \mathbf{u}^{[k]}+\underbrace{\left[\begin{array}{c}
\mathbf{h}^{[k]}(1)^{T} \sum_{k^{\prime} \neq k} \mathbf{u}^{\left[k^{\prime}\right]} \\
\vdots \\
\left.\mathbf{h}^{[k]}(M-1)^{T} \sum_{k^{\prime} \neq k} \mathbf{u}^{\left[k^{\prime}\right]}\right]
\end{array}\right]}_{\text {interference }}
$$

where $\mathbf{H}^{[k]} \in \mathbb{C}^{M \times M}$ is a full-rank matrix, $\mathbf{u}^{[k]} \in \mathbb{C}^{M \times 1}$ and, for simplicity, the temporal index refers to the position of the symbol extension in the alignment block instead of its position in the supersymbol. In the BIA scheme of [7], the interference term in (6) can be removed by measuring it in appropriate slots of Block 2. Then as long as the $\left\{\mathbf{h}^{[k]}(m)\right\}_{m=1}^{M}$ are linearly independent, the $M$ data streams $\mathbf{u}^{[k]}$ can be decoded by inverting the resulting linear system $\tilde{\mathbf{Y}}^{[k]}=\mathbf{H}^{[k]} \mathbf{u}^{[k]}$, where $\tilde{\mathbf{Y}}^{[k]}$ is the received signal after interference subtraction.

Since each of the $K_{t o t}$ users achieves $M$ DoF in each of its $(M-1)^{K_{t o t}-1}$ alignment blocks, which are distributed over a supersymbol of $(M-1)^{K_{t o t}}+K_{t o t}(M-1)^{K_{t o t}-1}$ symbol extensions, the sum DoF per symbol extension of cBIA is

$$
\mathrm{DoF}_{\mathrm{cBIA}}=\frac{M K_{t o t}}{M+K_{t o t}-1},
$$

where $K_{t o t}=\sum_{n=1}^{N_{B S}} K_{p, n}+K_{s h}$. For the symmetric scenario for which $N_{t, n}=N_{t}$ and $K_{p, n}=K_{p}$ for all $n$, (7) reduces to

$$
\operatorname{DoF}_{\mathrm{cBIA}, \text { symm }}=\frac{N_{B S} N_{t}\left(N_{B S} K_{p}+K_{s h}\right)}{N_{B S} N_{t}+N_{B S} K_{p}+K_{s h}-1} .
$$

For illustrative purposes, we consider the toy example shown in Fig. 3, where each BS is equipped with $N_{t}=2$ antennas. Each cell contains $K_{p}=1$ private user, whereas $K_{s h}=1$ shared user is located in the inter-cell area. Hence, the system has a total of $K_{t o t}=2 K_{p}+K_{s h}=3$ users. For this setting, cBIA achieves 2 DoF per symbol extension by employing a supersymbol comprising 54 symbol extensions.

\section{B. Moving to partially connected networks}

The cBIA scheme relies on the assumption of full connectivity, which does not hold in a typical cellular system. By reinstating the assumption that private users only receive signals 


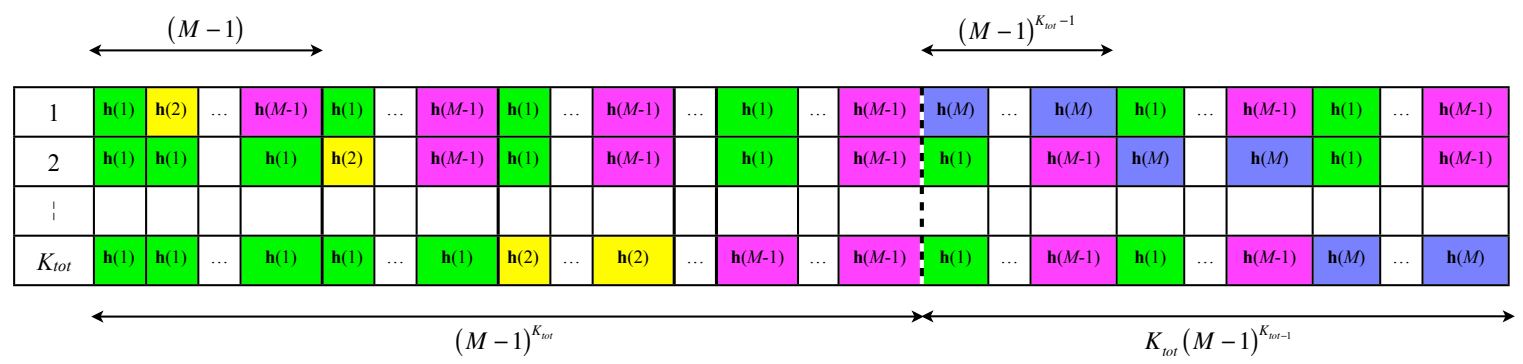

Fig. 2. Supersymbol for the cBIA scheme applied to $M=\sum_{n=1}^{N_{B S}} N_{t, n}$ antennas serving $K_{t o t}$ users.
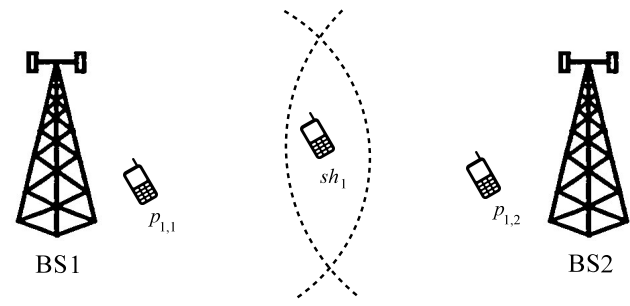

Fig. 3. Toy example: downlink scenario with full connectivity. The BSs are equipped with $N_{t}=2$ antennas each, and serve $K_{t o t}=3$ users.

from their respective BS, the DoF in (7) are no longer achievable if cBIA is applied. In a scenario with partial connectivity, as the one shown in Fig. 1, the channel between the BSs and private user $p_{k, n}$ at cell $n$ can be approximated as shown in (3), i.e, $\mathbf{h}^{\left[p_{k, n}\right]}(m) \approx\left[\begin{array}{lll}\mathbf{0}_{a, 1}{ }^{T} & \mathbf{h}^{\left[p_{k, n}, n\right]}(m)^{T} & \mathbf{0}_{b, 1}{ }^{T}\end{array}\right]^{T}$. Consequently, the channel matrix

$$
\mathbf{H}^{\left[p_{k, n}\right]}=\left[\mathbf{h}^{\left[p_{k, n}\right]}(1) \quad \cdots \quad \mathbf{h}^{\left[p_{k, n}\right]}(M)\right]^{T}
$$

corresponding to private user $p_{k, n}$ is no longer full-rank. Because of this, in (6) private user $p_{k, n}$ cannot decode the data streams sent by BSs $n^{\prime}, n^{\prime} \neq n$. Therefore, even if full data sharing is allowed between the BSs, cBIA fails to achieve the DoF given by (7) because of the lack of full connectivity.

Two questions that arise naturally are whether it is possible to devise a scheme that works in a scenario with partial connectivity and $M$ transmitters and what the achievable DoF are. In this paper we propose a network BIA scheme (nBIA) that not only allows the application of BIA to partially connected networks, but also attains more DoF than (7). To achieve this, we leverage the partial connectivity as a resource that allows to obtain more DoF, and decrease the number of modes used by the private users as well as the length of the supersymbol, which is one of the major limitations when applying cBIA in practical systems.

\section{A Network Blind Interference Alignment SCHEME FOR PARTIALLY CONNECTED CELLULAR NETWORKS}

In this section, we present the network BIA (nBIA) scheme for partially connected networks. We first describe the key idea of nBIA using the toy example of Section III. Then, for the sake of an easy exposition we describe nBIA for the symmetric scenario with $N_{B S}$ BSs, each equipped with $N_{t}$ antennas serving $K_{p}$ private users and $K_{s h}$ shared users.

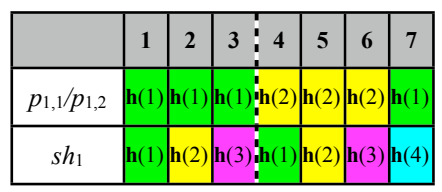

Fig. 4. Supersymbol of the nBIA scheme for the toy example. $N_{t}=2$, $K_{s h}=1$, and $K_{p, 1}=K_{p, 2}=1$.

\section{A. The key to Blind Interference Alignment in cellular systems}

Consider again the toy example of Fig. 3. This time, as shown in the figure, partial connectivity is assumed. The shared user receive data from both BS. On the other hand, each private user, i.e. $p_{1,1}$ and $p_{1,2}$, can only be served by its corresponding BS, BS 1 and BS 2, respectively. Thus, user $p_{k, n}$ does not decode the data sent by any other BS $n^{\prime}, n \neq n^{\prime}$. As a positive counterpart of this lack of connectivity, private users of a given BS are not subject to interference by any other $\mathrm{BS}$, at least in theory.

Since cBIA does not take into account the lack of full connectivity, it does not achieve (8). For the toy example, cBIA achieves $\frac{4}{3}$ DoF when there is partial connectivity, which is less than the $2 \mathrm{DoF}$ attained in a fully connected system. As an alternative to cBIA, consider the supersymbol of Fig. 4 and the beamforming matrices

$$
\mathbf{X}=\underbrace{\left[\begin{array}{lll}
\mathbf{I} & \mathbf{0} & \mathbf{0} \\
\mathbf{0} & \mathbf{I} & \mathbf{0} \\
\mathbf{0} & \mathbf{0} & \mathbf{I} \\
\mathbf{I} & \mathbf{0} & \mathbf{0} \\
\mathbf{0} & \mathbf{I} & \mathbf{0} \\
\mathbf{0} & \mathbf{0} & \mathbf{I} \\
\mathbf{0} & \mathbf{0} & \mathbf{0}
\end{array}\right]}_{p_{1,1}}\left[\begin{array}{l}
\mathbf{u}_{1}^{\left[p_{1,1}\right]} \\
\mathbf{u}_{2}^{\left[p_{1,1}\right]} \\
\left.\mathbf{u}_{3}^{\left[p_{1,1}\right]}\right]
\end{array}\right]+\underbrace{\left[\begin{array}{lll}
\mathbf{I} & \mathbf{0} & \mathbf{0} \\
\mathbf{0} & \mathbf{I} & \mathbf{0} \\
\mathbf{0} & \mathbf{0} & \mathbf{I} \\
\mathbf{I} & \mathbf{0} & \mathbf{0} \\
\mathbf{0} & \mathbf{I} & \mathbf{0} \\
\mathbf{0} & \mathbf{0} & \mathbf{I} \\
\mathbf{0} & \mathbf{0} & \mathbf{0}
\end{array}\right]}_{p_{1,2}}[\begin{array}{l}
\mathbf{u}_{1}^{\left[p_{1,2}\right]} \\
\mathbf{u}_{2}^{\left[p_{1,2}\right]} \\
\left.\mathbf{u}_{3}^{\left[p_{1,2}\right]}\right]
\end{array}+\underbrace{\left[\begin{array}{l}
\mathbf{I} \\
\mathbf{I} \\
\mathbf{I} \\
\mathbf{0} \\
\mathbf{0} \\
\mathbf{0} \\
\mathbf{I}
\end{array}\right]}_{s h_{1}} \mathbf{u}_{1}^{\left[s h_{1}\right],},
$$

where $\mathbf{x}[i] \in \mathbb{C}^{4 \times 1}, \mathbf{u}_{1}^{\left[s h_{1}\right]}=\left[\mathbf{u}_{1}^{\left[s h_{1}, 1\right]^{T}} \quad \mathbf{u}_{1}^{\left[s h_{1}, 2\right]^{T}}\right]^{T}$,

$$
\mathbf{u}_{\ell}^{\left[p_{1,1}\right]}=\left[\begin{array}{ll}
\mathbf{u}_{\ell}^{\left[p_{1,1}, 1\right]^{T}} & \mathbf{0}_{2,1}{ }^{T}
\end{array}\right]^{T}
$$

and $\mathbf{u}_{\ell}^{\left[p_{1,2}\right]}=\left[\begin{array}{ll}\mathbf{0}_{2,1} T & \left.\mathbf{u}_{\ell}^{\left[p_{1,2}, 2\right.}\right]^{T}\end{array}\right]^{T}$. The vectors $\mathbf{u}_{\ell}^{\left[p_{1, n}, n\right]} \in$ $\mathbb{C}^{2 \times 1}$ and $\mathbf{u}_{1}^{\left[s h_{1}, n\right]} \in \mathbb{C}^{2 \times 1}$ contain the symbols transmitted by BS $n$ to $p_{1, n}$ and $s h_{1}$, respectively, and $\mathbf{I}$ and $\mathbf{0}$ are the $4 \times 4$ identity and zero matrix, respectively.

Let us first focus on the transmission of data to shared user $s h_{1}$. As is explained in [7], since $s h_{1}$ is served by both BSs, 
to send $M=N_{t, 1}+N_{t, 2}=4$ distinguishable data streams, the BSs need to transmit $\mathbf{u}_{1}^{\left[s h_{1}\right]}$ repetitively during 4 symbol extensions over which the antenna of $s h_{1}$ switches through $M=4$ different modes. At the same time, the beams need to be aligned into one dimension at the users that are subject to interference by the signal sent to $s h_{1}$. Therefore, during these symbol extensions, $p_{1,1}$ and $p_{1,2}$ maintain the same mode. For instance, by looking at the supersymbol of Fig. 4 and the beamforming matrix of (10), we can check that symbol extensions 1, 2, 3 and 7 constitute an alignment block for $s h_{1}$. If we ignore the noise, the signal received at user $s h_{1}$ is

$$
\begin{aligned}
& {\left[\begin{array}{l}
\left.y^{\left[s h_{1}\right]}[1]\right] \\
y^{\left[s h_{1}\right]}[2] \\
y^{\left[s h_{1}\right]}[3] \\
y^{\left[s h_{1}\right]}[7]
\end{array}\right]=\left[\begin{array}{l}
\mathbf{h}^{\left[s h_{1}\right]}(1)^{T} \mathbf{x}[1] \\
\mathbf{h}^{\left[s h_{1}\right]}(2)^{T} \mathbf{x}[2] \\
\mathbf{h}^{\left[s h_{1}\right]}(3)^{T} \mathbf{x}[3] \\
\mathbf{h}^{\left[s h_{1}\right]}(4)^{T} \mathbf{x}[7]
\end{array}\right]} \\
& =\underbrace{\left[\begin{array}{l}
\mathbf{h}^{\left[s h_{1}\right]}(1)^{T} \\
\mathbf{h}^{\left[s h_{1}\right]}(2)^{T} \\
\mathbf{h}^{\left[s h_{1}\right]}(3)^{T} \\
\mathbf{h}^{\left[s h_{1}\right]}(4)^{T}
\end{array}\right.}_{\mathbf{H}^{\left[s h_{1}\right]}} \mathbf{u}_{1}^{\left[s h_{1}\right]}+\underbrace{\left[\begin{array}{l}
\mathbf{h}^{\left[s h_{1}\right]}(1)^{T}\left(\mathbf{u}_{1}^{\left[p_{1,1}\right]}+\mathbf{u}_{1}^{\left[p_{1,2}\right]}\right) \\
\mathbf{h}^{\left[s h_{1}\right]}(2)^{T}\left(\mathbf{u}_{2}^{\left[p_{1,1}\right]}+\mathbf{u}_{2}^{\left[p_{1,2}\right]}\right) \\
\mathbf{h}^{\left[s h_{1}\right]}(3)^{T}\left(\mathbf{u}_{3}^{\left[p_{1,1}\right]}+\mathbf{u}_{3}^{\left[p_{1,2}\right]}\right) \\
0
\end{array}\right]}_{\text {interference }}
\end{aligned}
$$

Since the channels $\mathbf{h}^{\left[s h_{1}\right]}(m), m \in\{1,2,3,4\}$ are generic, once the second term associated with the interference has been removed, user $s h_{1}$ can decode the 4 data streams $\mathbf{u}_{1}^{\left[s h_{1}\right]}$. Now, if we consider the signal received at the private users

$$
\begin{aligned}
& {\left[\begin{array}{l}
y^{\left[p_{1, n}\right]}[1] \\
y^{\left[p_{1, n}\right]}[2] \\
y^{\left[p_{1, n}\right]}[3] \\
y^{\left[p_{1, n}\right]}[7]
\end{array}\right]=\left[\begin{array}{l}
\mathbf{h}^{\left[p_{1, n}, n\right]}(1)^{T} \mathbf{x}^{[n]}[1]+\mathbf{0}_{2,1}^{T} \mathbf{x}^{\left[n^{\prime}\right]}[1] \\
\mathbf{h}^{\left[p_{1, n}, n\right]}(1)^{T} \mathbf{x}^{[n]}[2]+\mathbf{0}_{2,1}^{T} \mathbf{x}^{\left[n^{\prime}\right]}[2] \\
\mathbf{h}^{\left[p_{1, n}, n\right]}(1)^{T} \mathbf{x}^{[n]}[3]+\mathbf{0}_{2,1}^{T} \mathbf{x}^{\left[n^{\prime}\right]}[3] \\
\mathbf{h}^{\left[p_{1, n}, n\right]}(1)^{T} \mathbf{x}^{[n]}[7]+\mathbf{0}_{2,1}^{T} \mathbf{x}^{\left[n^{\prime}\right]}[7]
\end{array}\right]} \\
& =\underbrace{\left[\begin{array}{c}
\mathbf{h}^{\left[p_{1, n}, n\right]}(1)^{T} \mathbf{u}_{1}^{\left[p_{1, n}, n\right]} \\
\mathbf{h}^{\left[p_{1, n}, n\right]}(1)^{T} \mathbf{u}_{1, n}^{\left[p_{1, n}, n\right]} \\
\mathbf{h}^{\left[p_{1, n}, n\right]}(1)^{T} \mathbf{u}_{3}^{\left[p_{1, n}, n\right]}
\end{array}\right]}_{\text {desired signals }}+\underbrace{\left[\begin{array}{c}
\left.\mathbf{h}^{\left[p_{1, n}, n\right]}(1)^{T}\right] \\
\mathbf{h}^{\left[p_{1, n}, n\right]}(1)^{T} \\
\mathbf{h}^{\left[p_{1, n}, n\right]}(1)^{T} \\
\mathbf{h}^{\left[p_{1, n}, n\right]}(1)^{T}
\end{array}\right]}_{\text {interference }} \mathbf{u}_{1}^{\left[s h_{1}, n\right]},
\end{aligned}
$$

$n \in\{1,2\}, n^{\prime} \neq n$, we can observe that the four transmissions of $\mathbf{u}_{1}^{\left[s h_{1}\right]}=\left[\mathbf{u}_{1}^{\left[s h_{1}, 1\right]^{T}} \mathbf{u}_{1}^{\left[s h_{1}, 2\right]^{T}}\right]^{T}$ are aligned into one dimension at the private users. This way, since during symbol extension 7 BS $n$ only transmits $\mathbf{u}_{1}^{\left[s h_{1}, n\right]}$, by applying zero forcing based on $y^{\left[p_{1, n}\right]}[7], p_{1, n}$ can subtract the interference during symbol extensions 1,2 and 3.

Next, we concentrate on the transmission to private user $p_{1,1}$. Unlike shared user $s h_{1}, p_{1,1}$ can only be served by BS 1. To send $N_{t, 1}=2$ distinguishable symbols, $\mathbf{u}_{\ell}^{\left[p_{1,1}, 1\right]}$, to user $p_{1,1}$ in the absence of CSIT, BS 1 repeatedly transmitts $\mathbf{u}_{\ell}^{\left[p_{1,1}, 1\right]}$ during 2 symbol extensions over which the antenna of $p_{1,1}$ switches through $N_{t, 1}=2$ modes. Moreover, to align the two transmissions of $\mathbf{u}_{\ell}^{\left[p_{1,1}, 1\right]}$ into one dimension at the users subject to interference because of the transmission to user $p_{1,1}$, the affected users should keep the same radiation pattern. However, due to the partial connectivity of the network, $s h_{1}$ is now the only user subject to interference. Therefore, the radiation pattern of its antenna is the only one that has to be kept constant to project the interference caused by the transmissions of $\mathbf{u}_{\ell}^{\left[p_{1,1}, 1\right]}$ into one dimension. From the supersymbol of Fig. 4, we can easily check that the pairs of symbol extensions $\{1,4\},\{2,5\}$ and $\{3,6\}$ satisfy all the previous conditions. Each of these pairs constitutes an alignment block $\ell$ for private user $p_{1,1}$. For instance, consider the alignment block formed by symbol extensions $\{1,4\}$. Ignoring the noise, the signal received by the private user $p_{1,1}$ is

$$
\begin{gathered}
{\left[\begin{array}{l}
\left.y^{\left[p_{1,1}\right]}[1]\right] \\
y^{\left[p_{1,1}\right]}[4]
\end{array}\right]=\left[\begin{array}{l}
\left.\mathbf{h}^{\left[p_{1,1}, 1\right]}(1)^{T} \mathbf{x}^{[1]}[1]+\mathbf{0}_{2,1}^{T} \mathbf{x}^{[2]}[1]\right] \\
\mathbf{h}^{\left[p_{1,1}, 1\right]}(2)^{T} \mathbf{x}^{[1]}[4]+\mathbf{0}_{2,1}^{T} \mathbf{x}^{[2]}[4]
\end{array}\right]} \\
=\underbrace{\left[\begin{array}{l}
\mathbf{h}^{\left[p_{1,1}, 1\right]}(1)^{T} \\
\mathbf{h}^{\left[p_{1,1}, 1\right]}(2)^{T}
\end{array}\right]}_{\mathbf{H}^{\left[p_{1,1}, 1\right]}} \mathbf{u}_{1}^{\left[p_{1,1}, 1\right]}+\underbrace{\left[\begin{array}{c}
\left.\mathbf{h}^{\left[p_{1,1}, 1\right]}(1)^{T} \mathbf{u}_{1}^{\left[s h_{1}, 1\right]}\right] \\
0
\end{array}\right]}_{\text {interference }} .
\end{gathered}
$$

Private user $p_{1,1}$ applies zero forcing based on $y^{\left[p_{1,1}\right]}[7]$ to remove the interference at time instants 1, 2 and 3 (see (12)). Consequently, due to the fact that the channels $\mathbf{h}^{\left[p_{1,1}, 1\right]}(m)$, $m \in\{1,2\}$, are generic, the 2 symbols in $\mathbf{u}_{1}^{\left[p_{1,1}, 1\right]}$ can be decoded. The same procedure can be followed to decode the data steams $\mathbf{u}_{2}^{\left[p_{1,1}, 1\right]}$ and $\mathbf{u}_{3}^{\left[p_{1,1}, 1\right]}$ transmitted repetitively over the pairs of symbol extensions $\{2,5\}$ and $\{3,6\}$, respectively.

Recall that, as can be seen from (13), the transmission of data to private users of a specific cell does not cause interference to private users of other cells. Consequently, $p_{1,2}$ can reuse the same radiation pattern and the same beamforming matrix as $p_{1,1}$, as can also be verified from (10) and Fig. 4. This way, each pair of symbol extensions $\{1,4\}$, $\{2,5\}$ and $\{3,6\}$ also constitutes an alignment block of $p_{1,2}$. Moreover, note that in (11) the interference associated with the repeated transmissions of $\mathbf{u}_{\ell}^{\left[p_{1,2}, 2\right]}$ by BS 2 along the $\ell$ th alignment block of $p_{1,2}$ is aligned into the same single dimension as the transmissions of $\mathbf{u}_{\ell}^{\left[p_{1,1}, 1\right]}$ by BS 1 along the $\ell$-th alignment block of $p_{1,1}$. Hence, in (11) the interference term associated with the transmission of $\mathbf{u}_{\ell}^{\left[p_{1,1}, 1\right]}$ and $\mathbf{u}_{\ell}^{\left[p_{1,2}, 2\right]}$ can be removed if user $s h_{1}$ applies zero forcing based on the signals received during the time slot over which it is not receiving data. For instance, if $s h_{1}$ applies zero forcing based on $y^{\left[s h_{1}\right]}[4]=\mathbf{h}^{\left[s h_{1}\right]}(1)^{T}\left(\mathbf{u}_{1}^{\left[p_{1,1}\right]}+\mathbf{u}_{1}^{\left[p_{1,2}\right]}\right)$, it can remove all interference during symbol extension 1. Similarly, $s h_{1}$ can remove the interference during symbol extensions 2 and 3 by applying zero forcing based on $y^{\left[s h_{1}\right]}[5]$ and $y^{\left[s h_{1}\right]}[6]$, respectively.

In summary, using a reconfigurable antenna with $N_{t}=2$ modes, each private user achieves 6 DoF, 2 DoF per alignment block. At the same time, using a single antenna with 4 modes, shared user $s h_{1}$ achieves 4 DoF over only one alignment block. Therefore, a total of $16 \mathrm{DoF}$ are achieved along 7 symbol extensions, which yields $16 / 7 \mathrm{DoF}$ per symbol extension. Note that the new scheme improves upon the 2 DoF per symbol extension achieved by cBIA in a network where there is full connectivity and where all BSs share data intended to every user of the system. Furthermore, the improvement is achieved using a supersymbol of 7 instead of 54 symbol extensions.

To conclude, we note that the key of nBIA lies on the generalization of the definition of an alignment block to a communication system with partial connectivity. If a user $k$ 


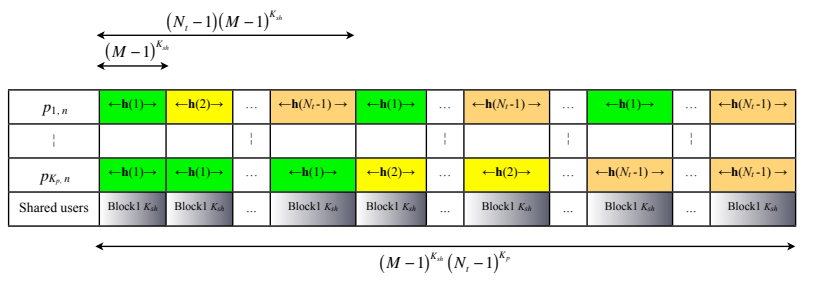

(a) S-Block 1 of the nBIA scheme for a symmetric cellular scenario with partial connectivity. Block1 $K_{s h}$ is shown in Fig. 6.

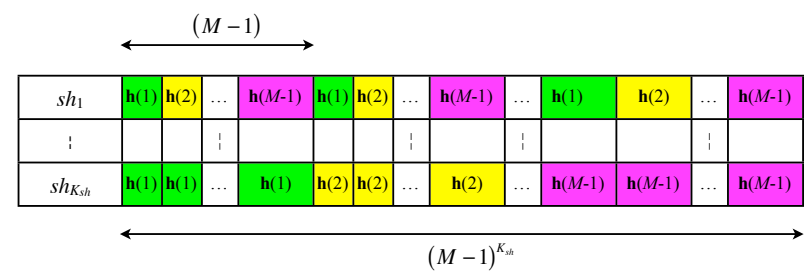

(b) Block 1 of the cBIA scheme for transmission to the $K_{s h}$ shared users.

Fig. 5. S-Block 1 of the nBIA scheme.

can be served by $N_{k}$ transmit antennas, then an alignment block for this user consists of $N_{k}$ symbol extensions over which it can receive $N_{k}$ distinguishable data streams. At the same time, these beams are only aligned into one dimension at all users subject to interference. On one hand, to decode $N_{k}$ distinguishable data streams, the channel state of user $k$ has to switch through $N_{k}$ different modes, one per symbol extension of the alignment block. As was seen in the toy example and as is described in [7], to align the aforementioned beams into one dimension at all users subject to interference, their channel state has to be maintained constant over the $N_{k}$ symbol extensions that form the alignment block of the desired user $k$. The data streams intended to a specific user need only be aligned into one dimension at those users where the power of the interference created by the aforementioned data streams is high enough, and therefore cannot be treated as noise.

\section{B. The network BIA scheme}

We now describe the nBIA scheme for the general symmetric scenario of a partially connected network. First, an sBIA scheme is implemented by each BS to send data to its set of $K_{p}$ private users. As shown in [7], this strategy allows each private user to remove interference caused by transmission to all other private users in its cell. The sBIA scheme is reused by all $N_{B S}$ BSs owing to the partial connectivity. Furthermore, all BSs of the system jointly implement a cBIA scheme to send data to the $K_{s h}$ shared users of the system and to let them cancel the interference among them. Finally, to obtain the supersymbol shown in Fig. 5(a), the two schemes are combined appropriately in order to remove the interference that the transmission of data to private users causes to the shared users and vice-versa.

1) Design of S-Block 1 of $n B I A$ : We first consider the design of Block 1 of the supersymbol of the nBIA scheme, which will be denoted as Super-Block 1 (S-Block 1). It comprises $(M-1)^{K_{s h}}\left(N_{t}-1\right)^{K_{p}}$ symbol extensions. As shown in Fig. 5(a), the symbol extensions of the shared users

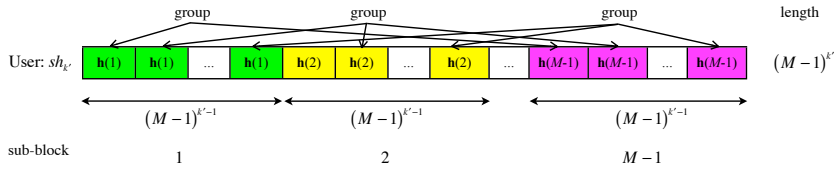

(a) Building block of shared user $s h_{k^{\prime}}$.

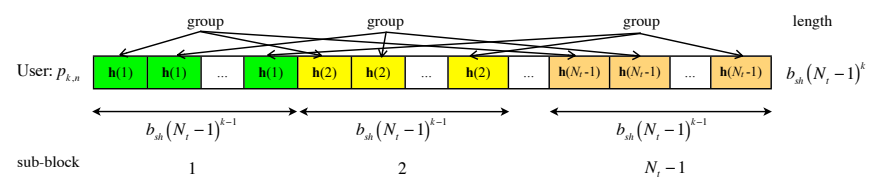

(b) Building block of private user $p_{k, n}$.

Fig. 6. Building blocks of the private and the shared users.

are formed concatenating $\left(N_{t}-1\right)^{K_{p}}$ Blocks 1 of a cBIA scheme for $K_{s h}$ users (see Fig. 5(b)). As plotted in Fig. 6(a), the building block of $s h_{k^{\prime}}$ is formed by $M-1$ sub-blocks comprising $(M-1)^{k^{\prime}-1}$ symbol extensions. During the $m$ th sub-block, $m \in\{1,2, \ldots, M-1\}$, the receiver of $s h_{k^{\prime}}$ maintains the $m$-th reconfigurable mode. Hence, the temporal correlation function of $s h_{k^{\prime}}$ in the entire S-Block 1 is

$$
f_{s h_{k^{\prime}}}(i)=\mathbf{h}^{\left[s h_{k^{\prime}}\right]}(m) \text { if } \bmod \left(i,(M-1)^{k^{\prime}}\right) \in \mathcal{I}_{s h}(m),
$$

with $i \in\left\{1,2, \ldots,(M-1)^{K_{s h}}\left(N_{t}-1\right)^{K_{p}}\right\}$, and

$$
\begin{aligned}
\mathcal{I}_{s h}(m)=\{ & (m-1)(M-1)^{k^{\prime}-1}+1 \\
& , \ldots, m(M-1)^{k^{\prime}-1}-1 \\
& \left., \bmod \left(m(M-1)^{k^{\prime}-1},(M-1)^{k^{\prime}}\right)\right\} .
\end{aligned}
$$

As can be seen in Fig. 5(a), Block-1 of the private users is closely based on Block 1 of a cBIA scheme aimed at transmitting to $K_{p}$ users using $N_{t}$ antennas. The mode of $p_{k, n}$ is periodic with the building block shown in Fig. 6(b), which is repeated $\left(N_{t}-1\right)^{K_{p}-k}$ times to form S-Block 1 . The building block is now composed of $N_{t}-1$ sub-blocks, each with length $b_{s h}\left(N_{t}-1\right)^{k-1}$, where $b_{s h}=(M-1)^{K_{s h}}$. As in the sub-blocks associated with $s h_{k^{\prime}}$, the $m$-th mode is used in the $m$-th subblock, $m \in\left\{1, \ldots, N_{t}-1\right\}$. This way, during each Block 1 of Fig. 5(b), each private user maintains a fixed mode. Hence, the temporal correlation function for private user $p_{k, n}$ for any cell $n \in\left\{1,2, \ldots, N_{B S}\right\}$ is

$$
f_{p_{k, n}}(i)=\mathbf{h}^{\left[p_{k, n}\right]}(m) \text { if } \bmod \left(i, b_{s h}\left(N_{t}-1\right)^{k}\right) \in \mathcal{I}_{p}(m)
$$

with $i \in\left\{1,2, \ldots,(M-1)^{K_{s h}}\left(N_{t}-1\right)^{K_{p}}\right\}$ and

$$
\begin{aligned}
\mathcal{I}_{p}(m)=\{ & (m-1) b_{s h}\left(N_{t}-1\right)^{k-1}+1 \\
& , \ldots, m b_{s h}\left(N_{t}-1\right)^{k-1}-1 \\
& \left., \bmod \left(m b_{s h}\left(N_{t}-1\right)^{k-1}, b_{s h}\left(N_{t}-1\right)^{k}\right)\right\} .
\end{aligned}
$$

For instance, in a two-cell scenario where $N_{t}=3, K_{p}=1$ and $K_{s h}=1$ S-Block 1 has the form shown in Fig. 7 .

2) Transmission strategy and beamforming matrices for $S$ Block 1: The key for the design of the beamforming matrices 


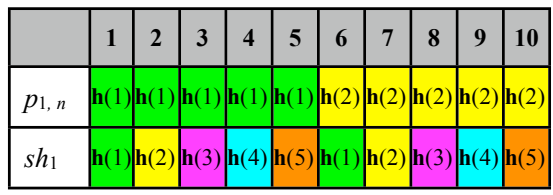

Fig. 7. Structure of S-Block 1 when $K_{p}=1$ and $K_{s h}=1$ in a two-cell scenario where each BS is equipped with $N_{t}=3$ antennas.

is to create alignment blocks that take into account the partial connectivity of the network. Each alignment block of a shared or private user corresponds to one block column in the corresponding beamforming matrix. Since each shared user $s h_{k^{\prime}}$ is served by all BSs, i.e. $M$ antennas, each block column of its beamforming matrix is obtained by placing an $M \times M$ identity matrix, $\mathbf{I}_{M}$, at the rows corresponding to the symbol extensions of the alignment block. The remaining blocks are filled with $M \times M$ zero matrices, $\mathbf{0}_{M}$. To obtain the signals transmitted from the BSs to shared user $s h_{k^{\prime}}$, the beamforming matrix is multiplied by

$$
\mathbf{u}_{\ell^{\prime}}^{\left[s h_{k^{\prime}}\right]}=\left[\begin{array}{lll}
\mathbf{u}_{\ell^{\prime}}^{\left[s h_{k^{\prime}}, 1\right]^{T}} & \ldots & \mathbf{u}_{\ell^{\prime}}^{\left[s h_{k^{\prime}}, n\right]^{T}} \ldots \\
\mathbf{u}_{\ell^{\prime}}^{\left[s h_{k^{\prime}}, N_{B S}\right]^{T}}
\end{array}\right]^{T}
$$

where $\mathbf{u}_{\ell^{\prime}}^{\left[s h_{k^{\prime}}\right]} \in \mathbb{C}^{N_{B S} N_{t} \times 1}, \quad \ell^{\prime}=1,2, \ldots,(M-$ $1)^{K_{s h}-1}\left(N_{t}-1\right)^{K_{p}}$ and $\mathbf{u}_{\ell^{\prime}}^{\left[s h_{k^{\prime}}, n\right]}$ contains the $N_{t}$ symbols transmitted from BS $n$ to $s h_{k^{\prime}}$ during alignment block $\ell^{\prime}$.

The same procedure is applied to obtain the beamfroming matrix for each private user at any cell $n$. However, $p_{k, n}$ is only served by the $N_{t}$ antennas of BS $n$. Recall that the signals $\mathbf{x}^{[n]}[i]$ transmitted by BSs $n$ do not contain data intended to any private user $p_{k, n^{\prime}}, n^{\prime} \neq n$. Therefore, each block column $\ell$ of the beamforming matrix is formed as for the shared users. However, to obtain the signals transmitted from the BSs to $p_{k, n}$ the corresponding beamforming matrix is multiplied by

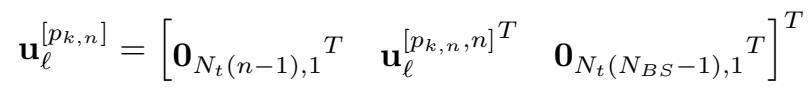

and $\ell=1,2, \ldots,\left(N_{t}-1\right)^{K_{p}-1}(M-1)^{K_{s h}}$.

To maintain the data beams of one alignment block distinguishable at the user for which they are intended, the channel between the transmit antennas and the user should change at each symbol extension of each alignment block. Moreover, during these symbol extensions, each of the affected users should maintain a constant channel so that interference be aligned. As is shown in Sections IV-B3 and IV-B4, in S-Block 1 both decodability and interference alignment requirements can be satisfied by using groups. Each group consists of the first $M-1$ or $N_{t}-1$ symbol extensions of the alignment block of a shared or private user, respectively. In particular, we can group the $\ell^{\prime}$-th symbol extension in each one of the $M-1$ sub-blocks within one building block as shown in Fig. 6(a) for shared user $s h_{k^{\prime}}$. Since each sub-block consists of $(M-1)^{k^{\prime}-1}$ symbol extensions, a total of $(M-1)^{k^{\prime}-1}$ groups can be built within one building block. As was mentioned above, each of these groups will be associated with a specific alignment block of $s h_{k^{\prime}}$. Similarly, as shown in Fig. 6(b), for private user $p_{k, n}$, the $\ell$-th symbol extension in each of the $N_{t}-1$ sub-blocks of one building block can be grouped. Since each sub-block of $p_{k, n}$ is now composed of $b_{s h}\left(N_{t}-1\right)^{k-1}$ symbol extensions, a total of $b_{s h}\left(N_{t}-1\right)^{k-1}$ groups can be formed within one building block. Recalling that S-Block 1 of $s h_{k^{\prime}}$ consists of $\left(N_{t}-1\right)^{K_{p}}(M-1)^{K_{s h}-k^{\prime}}$ building blocks of $(M-1)^{k^{\prime}}$ symbol extensions, the $\ell^{\prime}$-th group in the $p^{\prime}$-th building block of $s h_{k^{\prime}}$ comprises symbol extensions

$$
\left\{\left(p^{\prime}-1\right)(M-1)^{k^{\prime}}+\kappa(M-1)^{k^{\prime}-1}+\ell^{\prime}\right\}_{\kappa=0}^{M-2}
$$

where $\ell^{\prime} \in\left\{1,2, \ldots,(M-1)^{k^{\prime}-1}\right\}$ and

$$
p^{\prime} \in\left\{1,2, \ldots,(M-1)^{K_{s h}-k^{\prime}}\left(N_{t}-1\right)^{K_{p}}\right\} .
$$

Analogously, taking into account that S-Block 1 of $p_{k, n}, n \in$ $\left\{1,2, \ldots, N_{B S}\right\}$ is formed by $\left(N_{t}-1\right)^{K_{p}-k}$ building blocks of $b_{s h}\left(N_{t}-1\right)^{k}$ symbol extensions, the $\ell$-th group in its $p$-th building block consists of symbol extensions

$$
\left\{(p-1) b_{s h}\left(N_{t}-1\right)^{k}+\kappa b_{s h}\left(N_{t}-1\right)^{k-1}+\ell\right\}_{\kappa=0}^{N_{t}-2},
$$

where $p \in\left\{1,2, \ldots,\left(N_{t}-1\right)^{K_{p}-k}\right\}$, and $\ell \in\left\{1, \ldots, b_{s h}\left(N_{t}-\right.\right.$ $\left.1)^{k-1}\right\}$.

For instance, particularizing to our illustrative scenario with 2 cells, $N_{t}=3, K_{p}=1$ and $K_{s h}=1$, during S-Block 1

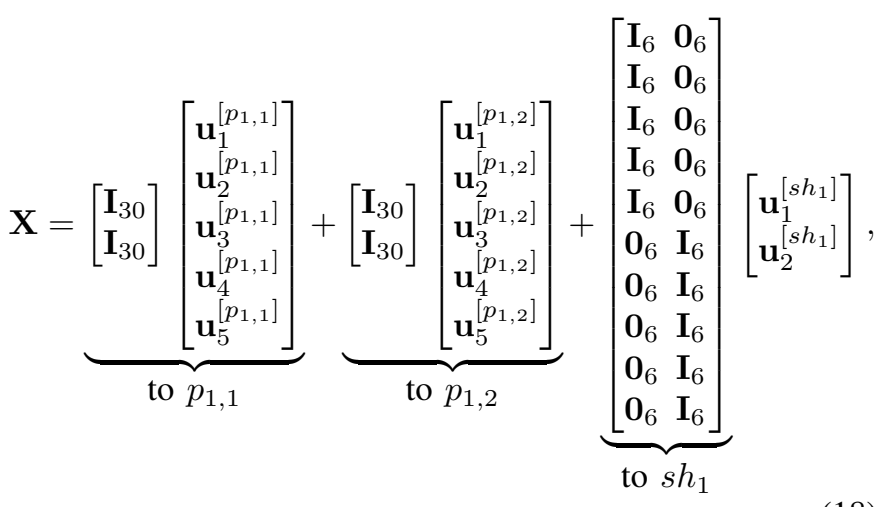

where $\mathbf{u}_{\ell}^{\left[p_{1,1}\right]}=\left[\begin{array}{ll}\left.\mathbf{u}_{\ell}^{\left[p_{1,1}, 1\right.}\right]^{T} & \mathbf{0}_{2,1}{ }^{T}\end{array}\right]^{T}, \quad \mathbf{u}_{\ell}^{\left[p_{1,2}\right]}=$ $\left[\begin{array}{ll}\mathbf{0}_{2,1}{ }^{T} & \mathbf{u}_{\ell}^{\left[p_{1,2}, 2\right]^{T}}\end{array}\right]^{T}$ and $\mathbf{u}_{\ell^{\prime}}^{\left[s h_{1}\right]}=\left[\begin{array}{ll}\mathbf{u}_{\ell^{\prime}}^{\left[s h_{1}, 1\right]^{T}} & \mathbf{u}_{\ell^{\prime}}^{\left[s h_{1}, 2\right]^{T}}\end{array}\right]^{T} \cdot$ Each private user has 5 groups formed by 2 symbol extensions. Specifically, for both private users these groups are formed by the pairs of symbol extensions $\{1,6\},\{2,7\}$, $\{3,8\},\{4,9\}$ and $\{5,10\}$. On the contrary, shared user $s h_{1}$ has two groups, each composed of 5 symbol extensions, i.e. $\{1,2,3,4,5\}$ and $\{6,7,8,9,10\}$.

3) Achieving decodability and interference alignment at the shared users: First, recall that the channel switching pattern for each shared user is created by concatenating $\left(N_{t}-1\right)^{K_{p}}$ identical Blocks 1 associated with a cBIA scheme aimed at transmitting data to $K_{s h}$ users. This way, based on the results in [7], it is straightforward to show that each group $\ell^{\prime}$ of each user $s h_{k^{\prime}}$ is formed by $M-1$ symbol extensions over which the mode of its antenna changes while the mode of all other shared users remains constant. Consequently, the data sent by all BSs to each user $s h_{k^{\prime}}$ over each of its alignment blocks can be decoded and the interference induced to the other shared users is aligned into one dimension of their signal space.

Note that the private users are also subject to interference 


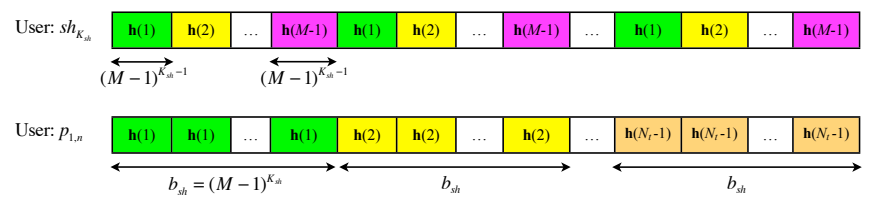

(a) The building blocks of shared users $s h_{K_{s h}}$ and $p_{1, n}$.

\begin{tabular}{|c|c|c|c|c|c|c|c|c|c|c|c|c|c|}
\hline User: $p_{k, n}$ & $\mathbf{h}(1)$ & $\mathbf{h}(2)$ & $\ldots$ & $\mathbf{h}\left(N_{t}-1\right)$ & $\mathbf{h}(1)$ & $\mathbf{h}(2)$ & $\ldots$ & $\mathbf{h}\left(N_{t}-1\right)$ & $\ldots$ & $\mathbf{h}(1)$ & $\mathbf{h}(2)$ & $\ldots$ & $\mathbf{h}\left(N_{t}-1\right)$ \\
\hline & $\vec{t}$ & & & $\stackrel{\left(N_{t}-1\right)^{\prime}}{\longrightarrow}$ & & & & & & & & & \\
\hline User: $p_{k+1, n}$ & $\mathbf{h}(1)$ & $\mathbf{h}(1)$ & $\ldots$ & $\mathbf{h}(1)$ & $\mathbf{h}(2)$ & $\mathbf{h}(2)$ & $\ldots$ & $\mathbf{h}(2)$ & $\ldots$ & $\mathbf{h}\left(N_{t}-1\right)$ & $\mathbf{h}\left(N_{t}-1\right)$ & $\ldots$ & $\mathbf{h}\left(N_{t}-1\right)$ \\
\hline
\end{tabular}

(b) The building blocks of private users $p_{k, n}$ and $p_{k+1, n}$.

Fig. 8. Building blocks of the private and shared users.

because of the data sent by the BSs to the shared users. To also align this interference, the $M-1$ data streams sent to a shared user over one of its groups also need to be contained into one dimension at all private users. As is also shown in Figs. 5(a) and 8(a), the channel mode of all private users does not change during an entire Block 1 of shared users. Moreover, recall that each group of shared user $s h_{k^{\prime}}$ is composed of symbols within a specific building block, which belongs to one of the Blocks 1 of $s h_{k^{\prime}}$. Hence, within each group of any shared user $s h_{k^{\prime}}$ the reconfigurable modes of the antennas of all private users remain the same. In conclusion, the interference caused by transmission to $s h_{k^{\prime}}$ during each one of its groups is aligned into one dimension at all private users.

4) Achieving decodability and interference alignment at the private users: We now concentrate on the private users. First, we check that the channel state of each private user changes at each symbol extension within any of its groups. Note that (17) specifies the symbol extensions of the $\ell$-th group in the $p$-th building block of private user $p_{k, n}$. Now, it can be easily seen that, for all $p \in\left\{1,2, \ldots,\left(N_{t}-1\right)^{K_{p}-k}\right\}$, the modulus of these symbol extensions with $b_{s h}\left(N_{t}-1\right)^{k}$ yields

$$
\left\{\kappa b_{s h}\left(N_{t}-1\right)^{k-1}+\ell\right\}_{\kappa=0}^{N_{t}-2}
$$

with $\ell \in\left\{1,2, \ldots, b_{s h}\left(N_{t}-1\right)^{k-1}\right\}$. Hence, from (15), the channel states of $p_{k, n}$ are $\mathbf{h}^{\left[p_{k, n}\right]}(1), \mathbf{h}^{\left[p_{k, n}\right]}(2), \ldots, \mathbf{h}^{\left[p_{k, n}\right]}\left(N_{t}-1\right)$ during the symbol extensions that form each one of its groups.

Next, we focus on proving that the interference caused by the transmission to private user $p_{k, n}$ is aligned into one dimension at the signal space of the other private users in cell $n$. First, consider private users $\left\{p_{j, n}\right\}_{j=1}^{k-1}$. Note that the remainder of the division of the symbol extensions in (17) by $b_{s h}\left(N_{t}-1\right)^{j}$ is the same, i.e. $\bmod \left(\ell, b_{s h}\left(N_{t}-1\right)^{j}\right)$, for a specific group $\ell$ in the $p$-th building block of $p_{k, n}$ and any $j \in\{1,2, \ldots, k-1\}$. Hence, from (15), within each group of $p_{k, n}$, the channel state of all other private users $\left\{p_{j, n}\right\}_{j=1}^{k-1}$ remains constant. Now, consider private users $\left\{p_{j, n}\right\}_{j=k+1}^{K_{p}}$. Notice that the lengths of the sub-blocks of the private users in S-Block 1 are larger than $b_{s h}\left(N_{t}-1\right)^{k}$, i.e. the length of a building block associated with private user $p_{k, n}$. Hence, since the boundaries of the building blocks of $p_{k, n}$ are aligned with those of the sub-blocks of $p_{j, n}, j \in\left\{k+1, k+2, \ldots, K_{p}\right\}$ (see Fig. 8(b)), the channels of this last sub-group of private users are the same within each group of $p_{k, n}$. Therefore, from the structure of S-Block 1 we can conclude that the data streams transmitted over the $N_{t}-1$ symbol extensions of the $\ell$-th group of user $p_{k, n}$ are aligned into one dimension at all other private users of cell $n$.

Ultimately, we show that interference caused by transmission to user $p_{k, n}$ is also aligned at the private users of the other cells $n^{\prime}, n^{\prime} \neq n$ as well as at the $K_{s h}$ shared users. Due to partial connectivity, we only need to verify that for each group of users $p_{k, n}$ the channel state of all shared users remains constant. Consider any shared user $s h_{k^{\prime}}$ and the symbol extensions in (17), which form the $\ell$-th group in the $p$-th building block of $p_{k, n}$. Since $b_{s h}$ is an integer multiple of $(M-1)^{k^{\prime}}$, the remainders of the indices of the symbol extensions in (17) divided by $(M-1)^{k^{\prime}}$ are the same, i.e. $\bmod \left(\ell,(M-1)^{k^{\prime}}\right)$. Consequently, from (14), within each group of $p_{k, n}$, the channel state of any user $s h_{k^{\prime}}$ is constant. Hence, the requirements of decodability and alignment are satisfied in each group of each private user.

As explained previously, the transmission of data from BS $n^{\prime}, n^{\prime} \neq n$, to its private users does not impose any constraints on the design of the channel pattern and the beamforming of private user $p_{k, n}$. Thus, private users $\left\{p_{k, n}\right\}_{n=1}^{N_{B S}}$ can reuse the same beamforming matrix and the same channel pattern in $\mathrm{S}$ Block 1 when receiving data from their corresponding BSs. This can be seen in our illustrative scenario in (18) and Fig. 7. More generally, the same fact can be verified in Fig. 5(a) and in (17) where the symbol extensions of the groups associated with private users $\left\{p_{k, n}\right\}_{n=1}^{N_{B S}}$ are the same. As a result, not only are the $N_{t}$ data beams transmitted within each group of one private user $p_{k, n}$ aligned into one dimension at each shared user, but also all data beams transmitted to all private users $\left\{p_{k, n}\right\}_{n=1}^{N_{B S}}$ within each group specified in (17) are projected into the same single dimension at each shared user.

5) Design of S-Block 2: From the design of S-Block-1 and the corresponding beamforming matrices, we can undertake the design of the switching pattern of all users during Block 2 of the nBIA scheme, which will be called Super-Block 2 (S-Block 2). The purpose of S-Block 2 is to complete the alignment blocks of all users so that each user can decode the data received along its groups and cancel the interference caused by the transmission of data to other users during S-Block 1. From (16) notice that the number of alignment blocks associated with each shared user is equal to $(M-1)^{K_{s h}-1}\left(N_{t}-1\right)^{K_{p}}$. Consequently, to complete the alignment blocks of the $K_{s h}$ shared users, a total of

$$
t_{s h}=K_{s h}\left[(M-1)^{K_{s h-1}}\left(N_{t}-1\right)^{K_{p}}\right]
$$

symbol extensions are needed in S-Block 2. As shown in Fig. 9, these symbol extensions are $L_{S-B_{1}}+1, L_{S-B_{1}}+$ $2, \ldots, L_{S-B_{1}}+t_{s h}$, where $L_{S-B_{1}}=(M-1)^{K_{s h}}\left(N_{t}-1\right)^{K_{p}}$ is the length of S-Block 1 . Within the aforementioned symbol extensions, sub-block

$$
\left\{L_{S-B_{1}}+\left(k^{\prime}-1\right) t_{s h} / K_{s h}+\ell^{\prime}\right\}_{\ell^{\prime}=1}^{t_{s h} / K_{s h}}
$$




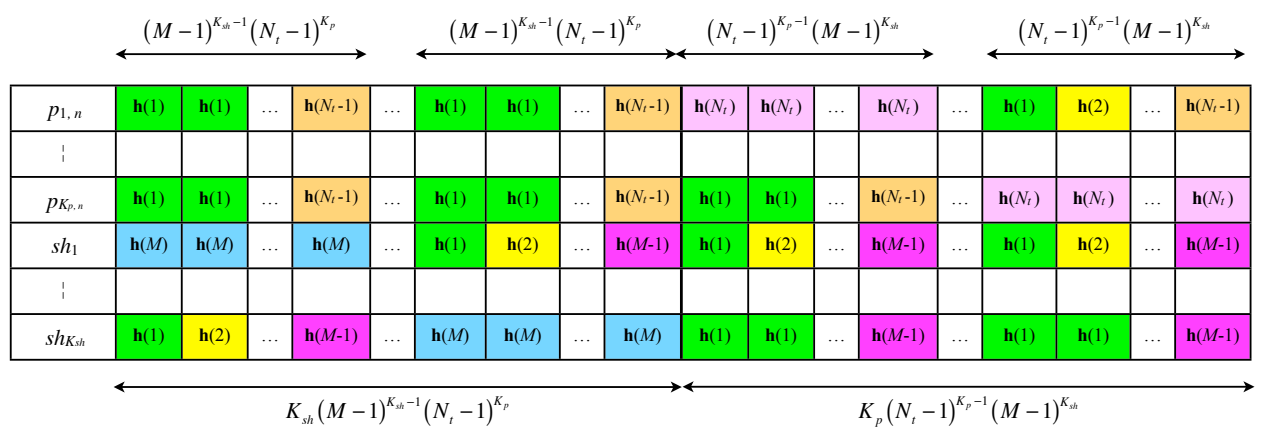

Fig. 9. S-Block 2 of the nBIA supersymbol.

with $k^{\prime} \in\left\{1,2, \ldots, K_{s h}\right\}$, provides the last symbol extensions of the alignment blocks of $s h_{k^{\prime}}$. In particular, each symbol extension specified in (21) constitutes the last element of the $\ell^{\prime}$-th alignment block of $s h_{k^{\prime}}$. Hence, in order to be able to decode the signals of interest over the alignment block, user $s h_{k^{\prime}}$ employs the $M$-th preset mode during each symbol extension in (21). This way, if the $N_{B S}$ BSs repetitively transmit $\mathbf{u}_{\ell^{\prime}}^{\left[s h_{k^{\prime}}\right]} \in \mathbb{C}^{M \times 1}$ within each symbol extension of the $\ell^{\prime}$-th alignment block of $s h_{k^{\prime}}$, the user can decode $\mathbf{u}_{\ell^{\prime}}^{\left[s h_{k^{\prime}}\right]}$ after removing the interference.

Since the interference caused by the first $M-1$ transmissions of $\mathbf{u}_{\ell^{\prime}}^{\left[s h_{k^{\prime}}\right]}$ during the $\ell^{\prime}$-th group of $s h_{k^{\prime}}$ in S-Block 1 is aligned into one dimension at all other shared and private users, zero forcing can be applied to remove it. Due to the fact that only $\mathbf{u}_{\ell^{\prime}}^{\left[s h_{k^{\prime}}\right]}, \ell^{\prime} \in\left\{1,2, \ldots, \frac{t_{s h}}{K_{s h}}\right\}$, is transmitted during each symbol extension of (21), any shared user $s h_{j^{\prime}} \neq s h_{k^{\prime}}$ and all private users $p_{k, n}$ can measure the interference caused by the transmission of $\mathbf{u}_{\ell^{\prime}}^{\left[s h_{k^{\prime}}\right]}$. Therefore, they can subtract the interference received in S-Block 1 if, during the symbol extensions given in (21), they maintain the same channel state as the one used during the $\ell^{\prime}$-th alignment group of $s h_{k^{\prime}}$. From (16) notice that the symbol extensions that form the $\ell^{\prime}$-th group of shared user $s h_{k^{\prime}}$ are

$$
\left\{p_{s h}\left(\ell^{\prime}, k^{\prime}\right)(M-1)^{k^{\prime}}+\kappa(M-1)^{k^{\prime}-1}+l_{s h}\left(\ell^{\prime}, k^{\prime}\right)\right\}_{\kappa=0}^{M-2}
$$

where

$$
l_{s h}\left(\ell, k^{\prime}\right)=\bmod \left(\ell^{\prime}-1,(M-1)^{k^{\prime}-1}\right)+1
$$

and $p_{s h}\left(\ell^{\prime}, k^{\prime}\right)=\left\lfloor\frac{\ell^{\prime}-1}{(M-1)^{k^{\prime}-1}}\right\rfloor$. Consequently, during the $\ell^{\prime}$-th symbol extension specified in (21) the channel state of shared users $s h_{j^{\prime}} \neq s h_{k^{\prime}}$ equals

$$
f_{s h_{j^{\prime}}}\left(p_{s h}\left(\ell^{\prime}, k^{\prime}\right)(M-1)^{k^{\prime}}+l_{s h}\left(\ell^{\prime}, k^{\prime}\right)\right),
$$

whereas the channel state for all private users $\left\{p_{k, n}\right\}_{k=1}^{N_{B S}}$ is

$$
f_{p_{k, n}}\left(p_{s h}\left(\ell^{\prime}, k^{\prime}\right)(M-1)^{k^{\prime}}+l_{s h}\left(\ell^{\prime}, k^{\prime}\right)\right),
$$

with $f_{s h_{j^{\prime}}}$ and $f_{p_{k, n}}$ given in (14) and (15), respectively.

Next, we consider the design of S-Block 2 for the private users. As we have seen in (17), the number of alignment blocks per private user equals $b_{s h}\left(N_{t}-1\right)^{K_{p}-1}$. Due to the partial connectivity, BSs $n$ and $n^{\prime}$ can transmit simultaneously the data associated with a specific alignment block of $p_{k, n}$ and $p_{k, n^{\prime}}$, respectively, without interfering with each other. Thus, one symbol extension of S-Block 2 can be reused by private users $\left\{p_{k, n}\right\}_{n=1}^{N_{B S}}$ to complete one of their alignment blocks. Thus, since there are $K_{p}$ private users per cell, a total of

$$
t_{p}=K_{p}\left[(M-1)^{K_{s h}}\left(N_{t}-1\right)^{K_{p}-1}\right]
$$

symbol extensions are needed in S-Block 2 for all private users. In order not to create any interference, similar to the symbol extensions of S-Block 2 for the shared users, each BS $n$ only transmits data to one specific user in its cell. However, this time the BSs do not transmit data to a specific shared user. Instead, each BS $n$ only transmits data to a specific private user $p_{k, n}$ during each of the $t_{p}$ symbol extensions. As shown in Fig. 9, the $t_{p}$ symbol extensions of S-Block 2 are $L_{S-B_{1}}+t_{s h}+1, L_{S-B_{1}}+t_{s h}+2, \ldots, L_{S-B_{1}}+t_{s h}+t_{p}$. Within these symbol extensions, the sub-block

$$
\left\{L_{S-B_{1}}+t_{s h}+(k-1) t_{p} / K_{p}+\ell\right\}_{\ell=1}^{t_{p} / K_{p}}
$$

$k \in\left\{1,2, \ldots, K_{p}\right\}$, provides the last symbol extensions of the alignment blocks of private users $\left\{p_{k, n}\right\}_{n=1}^{N_{B S}}$. Hence, during each symbol extension in (24) the private users have to keep the $N_{t}$-th preset mode. This way, if each BS $n$ applies a repetition code to send $\mathbf{u}_{\ell}^{\left[p_{k, n}\right]} \in \mathbb{C}^{N_{t} \times 1}$ during each symbol extension within the $\ell$-th alignment block of $\left\{p_{k, n}\right\}_{n=1}^{N_{B S}}$, each user $p_{k, n}$ at any cell $n$ can use the signals received during its $\ell$-th alignment block to decode $\mathbf{u}_{\ell}^{\left[p_{k, n}\right]}$.

Continuing the design of the symbol extensions of S-Block 2 , notice that the simultaneous transmission of $\left\{\mathbf{u}_{\ell}^{\left[p_{k, n}\right]}\right\}_{n=1}^{N_{B S}}$ during the $\ell$-th group of private users $\left\{p_{k, n}\right\}_{n=1}^{N_{B S}}$ are aligned into the same single dimension of the signal space of each shared user $s h_{k^{\prime}}$. Hence, to remove the interference caused by these transmissions, $s h_{k^{\prime}}$ can apply zero forcing based on the interference signal measured in S-Block 2. To do so, the preset mode of $s h_{k^{\prime}}$ during the $\ell$-th symbol extension in (24) has to be equal to the mode of $s h_{k^{\prime}}$ during the $\ell$-th alignment group of private users $\left\{p_{k, n}\right\}_{n=1}^{N_{B S}}$, which consists of symbol extensions

$$
\left\{p_{b}(\ell, k) b_{s h}\left(N_{t}-1\right)^{k}+\kappa b_{s h}\left(N_{t}-1\right)^{k-1}+l_{p}(\ell, k)\right\}_{\kappa=0}^{N_{t}-2}
$$

with $l_{p}(\ell, k)=\bmod \left(\ell-1, b_{s h}\left(N_{t}-1\right)^{k-1}\right)+1$ and $p_{p}(\ell, k)=\left\lfloor\frac{\ell-1}{b_{s h}\left(N_{t}-1\right)^{k-1}}\right\rfloor$. Mathematically, during the $\ell$-th 
symbol extension in (24) the channel state of $s h_{k^{\prime}}$ equals $f_{s h_{k^{\prime}}}\left(p_{p}(\ell, k) b_{s h}\left(N_{t}-1\right)^{k}+l_{p}(\ell, k)\right)$, where $f_{s h_{k^{\prime}}}$ is given in (14).

Due to the fact that the transmitted $\mathbf{u}_{\ell}^{\left[p_{k, n}\right]}$ during the symbol extensions of S-Block 1 are aligned into one dimension at the signal space of any private user $p_{j, n} \neq p_{k, n}$, each private user of cell $n$ can apply the same technique as shared user $s h_{k^{\prime}}$ to remove the interference caused by the transmission of $\mathbf{u}_{\ell}^{\left[p_{k, n}\right]}$. Specifically, at private user $p_{j, n} \neq p_{k, n}$, the interference is removed by applying zero forcing based on the signal received during the $\ell$-th symbol extension in (24) with the mode of its antenna equal to $f_{p_{j, n}}\left(p_{p}(\ell, k) b_{s h}\left(N_{t}-1\right)^{k}+l_{p}(\ell, k)\right)$, where $f_{p_{j, n}}$ is given in (15). Finally, due to the partial connectivity, the transmission of $\mathbf{u}_{\ell}^{\left[p_{k, n}\right]}$ to any private user $p_{k, n}$ at cell $n$ does not interfere with the communication between BS $n^{\prime}$ and any user $p_{j, n^{\prime}}$ at cell $n^{\prime} \neq n$. As a result, users $p_{j, n^{\prime}} \neq p_{k^{\prime}, n}$ do not need to cancel the interference caused by the transmission of data to $p_{k, n}$ during S-Block 1 .

\section{Achievable Degrees of Freedom}

With the nBIA scheme, each shared user achieves $M$ DoF per alignment block, whereas each private user attains $N_{t}$ DoF per alignment block. Since the total number of alignment blocks of each shared user is equal to $t_{s h} / K_{s h}=(M-$ $1)^{K_{s h}-1}\left(N_{t}-1\right)^{K_{p}}$ in the supersymbol of the nBIA scheme, a total of $M t_{s h} / K_{s h}$ DoF per supersymbol are achieved for each shared user. Following a similar reasoning and recalling that each private user employs $(M-1)^{K_{s h}}\left(N_{t}-1\right)^{K_{p}-1}$ alignment blocks per supersymbol, a total $N_{t} t_{p} / K_{p}$ DoF are attained by each private user in a supersymbol. Thus, since the length of the supersymbol equals $L_{S-B 1}+L_{S-B 2}$ symbol extensions where $L_{S-B 2}=t_{s h}+t_{p}$ is the number of symbol extensions in S-Block 2 (see Figs. 5(a), 5(b) and 9), when nBIA is used for the symmetric scenario the achievable sum DoF per symbol extension are

$$
\begin{aligned}
\mathrm{DoF}_{\mathrm{nBIA}}=\frac{K_{s h} M \frac{t_{s h}}{K_{s h}}+N_{B S} K_{p} N_{t} \frac{t_{p}}{K_{p}}}{L_{S-B 1}+L_{S-B 2}} \\
=\frac{M\left[K_{s h}\left(N_{t}-1\right)+K_{p}(M-1)\right]}{(M-1)\left(N_{t}-1\right)+K_{s h}\left(N_{t}-1\right)+K_{p}(M-1)} .
\end{aligned}
$$

As will be shown in Section $\mathrm{V}$, the sum DoF per symbol extension achieved by nBIA for the symmetric scenario is equal to the information-theoretic sum-DoF outer bound.

Remark 1. An alternative design of the supersymbol of the nBIA scheme can also be obtained. As shown in Fig. 10, a Block 1 associated with an sBIA scheme aimed at transmitting data to $K_{p}$ users is repeated $(M-1)^{K_{s h}}$ times to construct $S$-Block 1 for the $K_{p}$ private users of each cell $n$. For the shared users, S-Block 1 is formed by augmenting the length of the sub-blocks that form Block 1 of a cBIA scheme for a system with $K_{s h}$ shared users and $M$ transmit antennas. This time, the length of the sub-block of a shared user equals $\left(N_{t}-\right.$ $1)^{K_{p}}(M-1)^{k^{\prime}-1}$ symbol extensions. Similarly to S-Block 2 of Fig. 9, the alternative design for S-Block 2 is obtained by completing the alignment blocks whose groups form S-Block 1 .

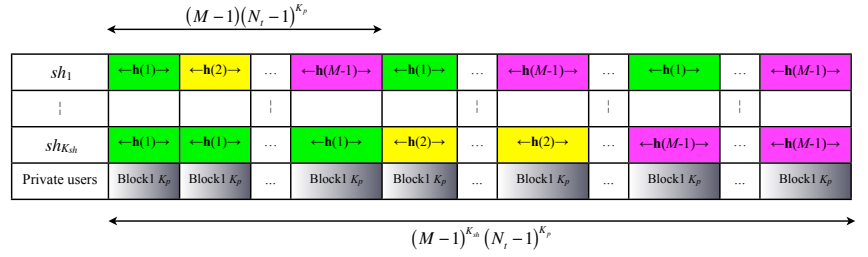

Fig. 10. Alternative design of S-Block 1 of the nBIA supersymbol.

It can be easily verified that the same DoF as in (26) can also be achieved by the alternative structure of the supersymbol.

\section{INFORMATION-THEORETIC SUM-DOF OUTER BOUND OF THE CELLULAR SCENARIO WITH PARTIAL CONNECTIVITY}

In this section we derive an outer bound for the sum DoF. The bound applies to the general partially connected network of Fig. 1, where the number of private users in each cell may be different. The proof is developed along the lines of [17]. In the symmetric case where the number of private users is the same in all cells, this bound is the same as the DoF that are achieved by the proposed nBIA scheme of Section IV; therefore, the scheme is DoF-optimal. For simplicity, the twocell scenario is considered. However, the proof can be easily extended to the case of $N_{B S}$ BSs.

Consider two BSs equipped with $N_{t, 1}$ and $N_{t, 2}$ antennas, which transmit to $K_{p, 1}$ and $K_{p, 2}$ private users, respectively, while $K_{s h}$ shared users are served simultaneously by both BSs. The messages and the rates of the users in cell $n \quad$ are denoted as $W^{\left[p_{1,1}, n\right]}, W^{\left[p_{2,1}, n\right]}, \ldots, W^{\left[p_{K_{p}, 1}, n\right]}$ and $\quad R^{\left[p_{1, n}\right]}, R^{\left[p_{2, n}\right]}, \ldots, R^{\left[p_{K_{p, n}}\right]}, \quad$ respectively; the messages and the rates of the shared users are denoted as $W^{\left[s h_{1}\right]}, W^{\left[s h_{2}\right]}, \ldots, W^{\left[s h_{K_{s h}}\right]}$ and $R^{\left[s h_{1}\right]}, R^{\left[s h_{2}\right]}, \ldots, R^{\left[s h_{K_{s h}}\right]}, \quad$ respectively. Accordingly, we express the sum rate as $R_{\Sigma}=R_{\Sigma p_{1}}+R_{\Sigma p_{2}}+R_{\Sigma s h}=$ $\sum_{n=1}^{2} \sum_{k=1}^{K_{p, n}} R^{\left[p_{k, n}\right]}+\sum_{k^{\prime}=1}^{K_{s h}} R^{\left[s h_{k^{\prime}}\right]}$. We also define the message sets

$$
\mathcal{W}^{\left[p_{n}\right]}=\left\{W^{\left[p_{1,2}, n\right]}, \ldots, W^{\left[p_{K_{p}, 2}, n\right]}\right\}
$$

with $n \in\{1,2\}$, and

$$
\mathcal{W}^{[s h]}=\left\{W^{\left[s h_{1}\right]}, \ldots, W^{\left[s h_{K_{s h}}\right]}\right\} .
$$

Consider private user $p_{1,1}$ in cell 1 , who desires message $W^{\left[p_{1,1}, 1\right]}$. In particular, consider $N_{t, 1}$ random realizations of this user, each corresponding to a different realization of the channel. Because there is no CSIT, and we require reliable decoding (probability of error approaching zero), each realization of the user should also have probability of error approaching zero. According to (1) the signal received by the $m$-th realization of user $p_{1,1}$ at time $i$ can be written as

$$
y_{m}^{\left[p_{1,1}\right]}[i]=\mathbf{h}_{m}^{\left[p_{1,1}, 1\right]^{T}} \mathbf{x}_{m}^{[1]}[i]+z_{m}^{\left[p_{1,1}\right]},
$$

where $m \in\left\{1,2, \ldots, N_{t, 1}\right\}$ and the i.i.d Gaussian noise terms have been normalized to have unit variance.

Applying Fano's inequality to codebooks spanning $n$ chan- 
nel uses, we have

$$
\begin{aligned}
n R^{\left[p_{1,1}\right]} \leq & I\left(W^{\left[p_{1,1}, 1\right]} ;\left(y_{m}^{\left[p_{1,1}\right]}\right)^{n}\right)+o(n) \\
= & h\left(\left(y_{m}^{\left[p_{1,1}\right]}\right)^{n}\right)-h\left(\left(y_{m}^{\left[p_{1,1}\right]}\right)^{n} \mid W^{\left[p_{1,1}, 1\right]}\right)+o(n) \\
\leq & n(\log (P)+o(\log (P))) \\
& -h\left(\left(y_{m}^{\left[p_{1,1}\right]}\right)^{n} \mid W^{\left[p_{1,1}, 1\right]}\right)+o(n),
\end{aligned}
$$

where $P$ is the total transmit power constraint at each BS. Since this is true for every $m \in\left\{1,2, \ldots, N_{t, 1}\right\}$, in (29) we add the inequalities corresponding to all $N_{t, 1}$ realizations. For steps (30)-(32), we use $h(A, B) \leq h(A)+h(B), h(A, B \mid C)=$ $h(A \mid B C)+h(B \mid C)$ and the independence between any pair of messages. To justify step (32)-(33), first note that from $\left[y_{1}^{\left[p_{1,1}\right]}, \ldots, y_{N_{t, 1}}^{\left[p_{1,1}\right]}\right]$ we have $N_{t, 1}$ linear equations in the $N_{t, 1}$ transmitted symbols $\mathbf{x}^{[1]}=\left[x_{1,1}^{[1]}, \ldots x_{1, N_{t, 1}}^{[1]}\right.$, each subject to additive noise whose variance does not depend on $P$. Since the channel realizations are random, these linear equations are almost surely linearly independent, i.e., one can recover $\mathbf{x}^{[1]}$ from these equations, subject to noise distortion. However, from $\mathbf{x}^{[1]}$ and noise the messages intended for the users in cell 1 that originate at BS 1 can be recovered. Thus, the remaining uncertainty is just due to noise, which is no more that $o(\log (P))$ per channel use. Moreover, in (32)-(33) we use the fact that conditioning cannot increase the entropy.

Proceeding similarly for private user $p_{1,2}$ in cell 2 ,

$$
\begin{aligned}
n N_{t, 2} R^{\left[p_{1,2}\right]} \leq & n N_{t, 2} \log (P)-n\left(R_{\Sigma p_{2}}-R^{\left[p_{1,2}\right]}\right) \\
& -h\left(\left(y_{1}^{\left[p_{1,2}\right]}, \ldots, y_{N_{t, 2}}^{\left[p_{1,2}\right]}\right)^{n} \mid \mathcal{W}^{\left[p_{1}\right]}, \mathcal{W}^{\left[p_{2}\right]}\right) \\
& +o(n)+n o(\log (P)) .
\end{aligned}
$$

Adding (32) and (34), we obtain (35)-(36). Step (35)(36) is justified as follows. From $\left(y_{1}^{\left[p_{1,1}\right]}, \ldots, y_{N_{t, 1}}^{\left[p_{1,1}\right]}\right)^{n}$ and $\left(y_{1}^{\left[p_{1,2}\right]}, \ldots, y_{N_{t, 2}}^{\left[p_{1,2}\right]}\right)^{n}$ we have $N_{t_{1}}+N_{t, 2}$ generic linear equations (subject to noise distortion), which are almost surely linearly independent and can therefore be solved to recover $N_{t, 1}+N_{t, 2}$ input symbols from both BSs, subject to noise distortion. Thus, we can recover all messages within an $n o(\log (P))$ term due to noise distortion. Moreover, we use $h(A, B) \leq h(A)+h(B)$.

Replacing $p_{1,1}$ and $p_{1,2}$ with any private users $p_{k, 1}$ and $p_{j, 2}$ in (36), respectively, after dividing by $n \log (P)$, taking first the limit $n \rightarrow \infty$ and then the limit $P \rightarrow \infty$, a rearranging of the terms yields the following DoF outer bound

$$
\left(N_{t, 1}-1\right) d^{\left[p_{k, 1}\right]}+\left(N_{t, 2}-1\right) d^{\left[p_{j, 2}\right]}+d_{\Sigma} \leq N_{t, 1}+N_{t, 2},
$$

where $d_{\Sigma}=d_{\Sigma s h}+d_{\Sigma p_{1}}+d_{\Sigma p_{2}}$. Adding all these bounds, after other rearrangement we obtain

$$
\begin{aligned}
& K_{p, 2}\left(K_{p, 1}+N_{t, 1}-1\right) d_{\Sigma p_{1}}+K_{p, 1}\left(K_{p, 2}+N_{t, 2}-1\right) d_{\Sigma p_{2}} \\
& \quad+K_{p, 1} K_{p, 2} d_{\Sigma s h} \leq K_{p, 1} K_{p, 2}\left(N_{t, 1}+N_{t, 2}\right) .
\end{aligned}
$$

Next, consider the first shared user, who wants the message $W^{\left[s h_{1}\right]}$. Also consider $M=N_{t, 1}+N_{t, 2}$ realizations of this user. For any realization $m$, starting from Fano's inequality, we go through a similar series of steps, as follows

$$
\begin{aligned}
n R^{\left[s h_{1}\right]} \leq & I\left(W^{\left[s h_{1}\right]} ;\left(y_{m}^{\left[s h_{1}\right]}\right)^{n}\right)+o(n) \\
\leq & n \log (P)-h\left(\left(y_{m}^{\left[s h_{1}\right]}\right)^{n} \mid W^{\left[s h_{1}\right]}\right) \\
& +o(n)+n o(\log (P)) .
\end{aligned}
$$

Adding the bounds for all $M$ realizations,

$$
\begin{aligned}
n M R^{\left[s h_{1}\right] \leq} & n M \log (P)-h\left(\left(y_{1}^{\left[s h_{1}\right]}, \ldots, y_{M}^{\left[s h_{1}\right]}\right)^{n} \mid W^{\left[s h_{1}\right]}\right) \\
& +o(n)+n o(\log (P)) \\
\leq & n M \log (P)-n\left(R_{\Sigma p_{1}}+R_{\Sigma p_{2}}+R_{\Sigma s h}-R^{\left[s h_{1}\right]}\right) \\
& +o(n)+n o(\log (P)) .
\end{aligned}
$$

Hence, we obtain the DoF outer bound

$$
(M-1) d^{\left[s h_{1}\right]}+d_{\Sigma p_{1}}+d_{\Sigma p_{2}}+d_{\Sigma s h} \leq M .
$$

If we now sum (41) over all shared users, we obtain

$$
\left(K_{s h}+M-1\right) d_{\Sigma s h}+K_{s h}\left(d_{\Sigma p_{1}}+d_{\Sigma p_{2}}\right) \leq K_{s h} M .
$$

The final DoF outer bounds that we need are (38) and (42). Specialized to the symmetric setting where $N_{t, 1}=N_{t, 2}=N_{t}$ and $K_{p, 1}=K_{p, 2}=K_{p}$, we have the sum-DoF outer bound

$\operatorname{maximize} \quad d_{\Sigma s h}+d_{\Sigma p_{1}}+d_{\Sigma p_{2}}$

subject to

$$
\begin{aligned}
& K_{p} d_{\Sigma s h}+\left(K_{p}+N_{t}-1\right)\left(d_{\Sigma p_{1}}+d_{\Sigma p_{2}}\right) \leq 2 K_{p} N_{t} \\
& \left(2 N_{t}+K_{s h}-1\right) d_{\Sigma s h}+K_{s h}\left(d_{\Sigma p_{1}}+d_{\Sigma p_{2}}\right) \leq 2 K_{s h} N_{t}
\end{aligned}
$$

This linear program is easily solved to obtain the sum-DoF bound

$$
d_{\Sigma} \leq \frac{M\left[K_{s h}\left(N_{t}-1\right)+K_{p}(M-1)\right]}{(M-1)\left(N_{t}-1\right)+K_{s h}\left(N_{t}-1\right)+K_{p}(M-1)},
$$

which is achieved when

$$
\begin{aligned}
d_{\Sigma p_{n}} & =\frac{N_{t} K_{p}(M-1)}{(M-1)\left(N_{t}-1\right)+K_{s h}\left(N_{t}-1\right)+K_{p}(M-1)} \\
d_{\Sigma s h} & =\frac{M K_{s h}\left(N_{t}-1\right)}{(M-1)\left(N_{t}-1\right)+K_{s h}\left(N_{t}-1\right)+K_{p}(M-1)} .
\end{aligned}
$$

Note that this is exactly the same DoF achieved by the nBIA scheme proposed in Section IV for symmetric cellular networks whereas the number of private users is the same at each cell.

\section{Asymmetric Partially Connected Cellular NETWORKS}

So far, a symmetric scenario has been considered. In this section, the nBIA scheme is extended to asymmetric cellular networks where the number of private users can be different at each cell. It will be shown that there exist some settings for which the proposed extension achieves the sum-DoF outer bound of Section V. However, this is not generally the case, and therefore, the DoF optimality of the proposed approach for asymmetric cellular networks is still an open problem. 


$$
\begin{aligned}
n N_{t, 1} R^{\left[p_{1,1}\right]} \leq & n N_{t, 1} \log (P)-\sum_{m=1}^{N_{t, 1}} h\left(\left(y_{m}^{\left[p_{1,1}\right]}\right)^{n} \mid W^{\left[p_{1,1}, 1\right]}\right)+o(n)+n o(\log (P)) \\
\leq & n N_{t, 1} \log (P)-h\left(\left(y_{1}^{\left[p_{1,1}\right]}, \ldots, y_{N_{t, 1}}^{\left[p_{1,1}\right]}\right)^{n} \mid W^{\left[p_{1,1}, 1\right]}\right)+o(n)+n o(\log (P)) \\
\leq & n N_{t, 1} \log (P)-h\left(W^{\left[p_{2,1}, 1\right]}, \ldots, W^{\left[p_{K_{p}, 1}, 1\right]},\left(y_{1}^{\left[p_{1,1}\right]}, \ldots, y_{N_{t, 1}}^{\left[p_{1,1}\right]}\right)^{n} \mid W^{\left[p_{1,1}, 1\right]}\right) \\
& +h\left(W^{\left[p_{2,1}, 1\right]}, \ldots, W^{\left[p_{K_{p}, 1}, 1\right]} \mid\left(y_{1}^{\left[p_{1,1}\right]}, \ldots, y_{N_{t, 1}}^{\left[p_{1,1}\right]}\right)^{n}, W^{\left[p_{1,1}, 1\right]}\right)+o(n)+n o(\log (P)) \\
\leq & n N_{t, 1} \log (P)-h\left(W^{\left[p_{2,1}, 1\right]}, \ldots, W^{\left[p_{K_{p}, 1}, 1\right]}\right)-h\left(\left(y_{1}^{\left[p_{1,1}\right]}, \ldots, y_{N_{t, 1}}^{\left[p_{1,1}\right]}\right)^{n} \mid \mathcal{W}^{\left[p_{1}\right]}\right) \\
& +\underbrace{h\left(W^{\left[p_{2,1}, 1\right]}, \ldots, W^{\left[p_{K_{p}, 1}, 1\right]} \mid\left(y_{1}^{\left[p_{1,1}\right]}, \ldots, y_{N_{t, 1}}^{\left[p_{1,1}\right]}\right)^{n}, W^{\left[p_{1,1}, 1\right]}\right)+o(n)+n o(\log (P))}_{\leq n o(\log (P))} \\
\leq & n N_{t, 1} \log (P)-n\left(R_{\Sigma p_{1}}-R^{\left[p_{1,1}\right]}\right)-h\left(\left(y_{1}^{\left[p_{1,1}\right]}, \ldots, y_{N_{t, 1}}^{\left[p_{1,1}\right]}\right)^{n} \mid \mathcal{W}^{\left[p_{1}\right]}, \mathcal{W}^{\left[p_{2}\right]}\right)+o(n)+n o(\log (P)) .
\end{aligned}
$$

$$
\begin{aligned}
n\left(N_{t, 1} R^{\left[p_{1,1}\right]}+N_{t, 2} R^{\left[p_{1,2}\right]}\right) \leq & n\left(N_{t, 1}+N_{t, 2}\right) \log (P)-n\left(R_{\Sigma p_{1}}+R_{\Sigma p_{2}}-R^{\left[p_{1,1}\right]}-R^{\left[p_{1,2}\right]}\right) \\
& -h\left(\left(y_{1}^{\left[p_{1,1}\right]}, \ldots, y_{N_{t, 1}}^{\left[p_{1,1}\right]}\right)^{n},\left(y_{1}^{\left[p_{1,2}\right]}, \ldots, y_{N_{t, 2}}^{\left[p_{1,2}\right]}\right)^{n} \mid \mathcal{W}^{\left[p_{1}\right]}, \mathcal{W}^{\left[p_{2}\right]}\right)+o(n)+n o(\log (P)) \\
\leq & n\left(N_{t, 1}+N_{t, 2}\right) \log (P)-n\left(R_{\Sigma p_{1}}+R_{\Sigma p_{2}}-R^{\left[p_{1,1}, 1\right]}-R^{\left[p_{1,2}, 2\right]}\right) \\
& -n R_{\Sigma s h}+o(n)+n o(\log (P)) .
\end{aligned}
$$

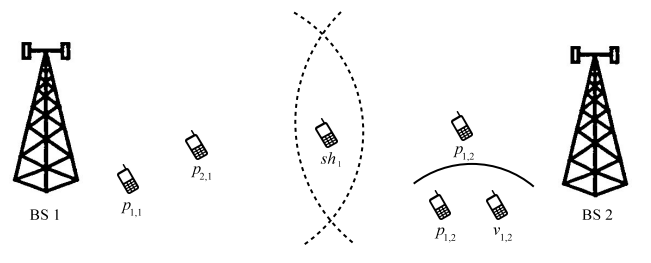

Fig. 11. Asymmetric toy example. BS 1 and BS 2 transmit to $K_{p, 1}=2$ and $K_{p, 2}=1$, respectively, and both transmit to $K_{s h}=1$ shared user.

For illustrative purposes, we consider a toy example where $N_{t}=2, K_{s h}=1, K_{p, 1}=2$ and $K_{p, 2}=1$ (see Fig. 11). By solving the optimization problem of (37) and (41), the outer bound is 2.5 DoF. The supersymbol of the nBIA scheme of Section IV is shown in Fig. 12. Each private user exploits 3 alignment blocks, which provide $N_{t}=2$ DoF each, whereas the shared user attains $M=4 \mathrm{DoF}$ during 10 symbol extensions. Therefore, the proposed scheme attains $\frac{22}{10} \mathrm{DoF}$ in total, which is $\frac{3}{10}$ below the outer bound.

In Fig. 12, the pairs of symbol extensions $\{1,4\},\{2,5\}$ and $\{3,6\}$ constitute alignment blocks of private user $p_{1,2}$. During each of the aforementioned alignment blocks, private user $p_{1,2}$ achieves 2 DoF. Moreover, symbol extension $\{10\}$ is used by $p_{1,2}$ to remove the interference caused by the transmission to the shared user $s h_{1}$. Note also that symbol extensions $\{7,8,9\}$, which are employed by BS 1 to transmit to $p_{2,1}$ and complete its alignment blocks, are used by shared user $s h_{1}$ in order to remove the interference caused by the transmission from BS 1 to user $p_{2,1}$. On the contrary, symbol extensions $\{7,8,9\}$ are idle for private user $p_{1,2}$ since it is not subject to interference caused by the transmission to $p_{2,1}$, and therefore, does not

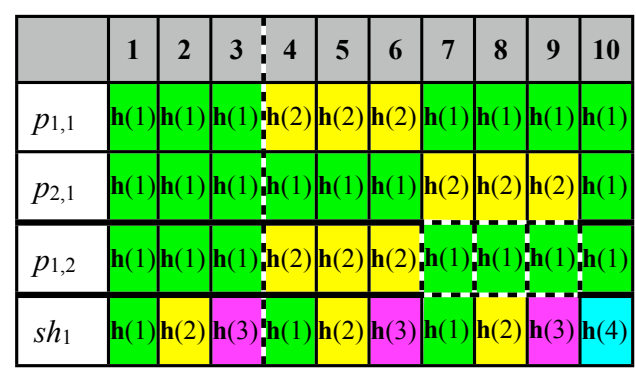

Fig. 12. Supersymbol for the asymmetric scenario with $N_{t}=2, K_{p, 1}=2$, $K_{p, 2}=1$ and $K_{s h}=1$. Dashed lines represent the idle slots that can be used for transmission of $v_{1,2}$.

need to remove it. We can devise a virtual user $v_{1,2}$, which is the same physical user as $p_{1,2}$. Since no changes are required at BS 1, we only consider transmission of BS 2 . Taking virtual user $v_{1,2}$ into account, the symbols sent by BS 2 are now

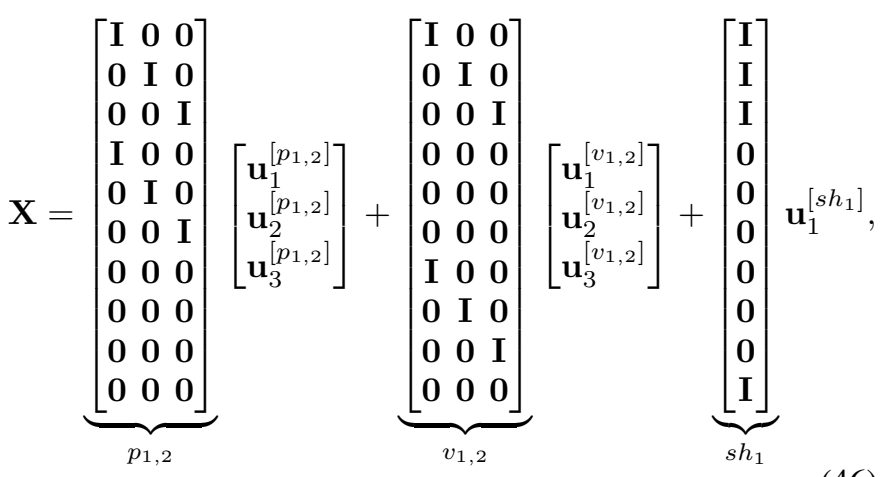

where $\quad \mathbf{u}_{1}^{\left[s h_{1}\right]}=\left[\begin{array}{ll}\mathbf{0}_{2,1}{ }^{T} & \left.\mathbf{u}_{1}^{\left[s h_{1}, 2\right.}\right]^{T}\end{array}\right]^{T}, \quad \mathbf{u}_{\ell}^{\left[p_{1,2}\right]}=$ 


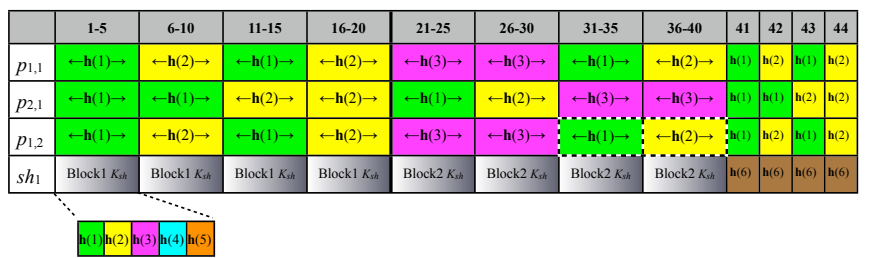

Fig. 13. Supersymbol for asymmetric scenario with $N_{t}=3, K_{p, 1}=2$, $K_{p, 2}=1$ and $K_{s h}=1$. Dashed lines represent the idle slots that can be used for transmission of $v_{1,2}$.

$\left.\left[\mathbf{0}_{2,1}{ }^{T} \quad \mathbf{u}_{\ell}^{\left[p_{1,2}, 2\right.}\right]^{T}\right]^{T}$, and $\mathbf{u}_{\ell}^{\left[v_{1,2}\right]}=\left[\mathbf{0}_{3,1}{ }^{T} u_{\ell}^{\left[p_{1,2}, 2\right]^{T}}\right]^{T}$.

The private users $p_{1,1}$ and $p_{2,1}$ served by $\mathrm{BS} 1$ are not subject to interference by the transmission of $\mathrm{BS} 2$ to $v_{1,2}$. The shared user can measure the sum of interference from transmission to both $p_{2,1}$ and $v_{1,2}$ during symbol extensions $\{7,8,9\}$ and remove it from symbol extensions $\{1,2,3\}$. Note that, since only the sum of the interference terms caused by the transmission to $v_{1,2}$ and $p_{2,1}$ can be measured, this scheme requires to retransmit the symbols $\left\{\mathbf{u}_{\ell}^{\left[v_{1,2}\right]}\right\}_{\ell=1}^{3}$ not only during symbol extensions $\{7,8,9\}$, but also during symbol extensions $\{1,2,3\}$, respectively. Furthermore, transmission of BS 2 to private user $p_{1,2}$ is carried out using the nBIA scheme of Section IV. However, private user $p_{1,2}$ is now subject to interference caused by the transmission from BS 2 to virtual user $v_{1,2}$ during symbol extensions $\{1,2,3\}$. In order to remove it, private user $p_{1,2}$ only needs to measure it during symbol extensions $\{7,8,9\}$ with the same channel mode as in symbol extensions $\{1,2,3\}$, respectively. After removing this interference, the DoF attained by $p_{1,2}$ are not affected.

During symbol extensions $\{7,8,9\}$, BS 2 only transmits to $v_{1,2}$. Additionally, due to the partial connectivity of the network, notice that virtual user $v_{1,2}$ is not subject to interference caused by the transmission from BS 1 to private user $p_{2,1}$. Hence, the virtual user attains 3 additional DoF, one per symbol extension. Since the supersymbol consists of 10 slots, an improvement of $\frac{3}{10} \mathrm{DoF}$ is achieved compared to the $\frac{22}{10}$ DoF achieved by the nBIA scheme of Section IV. As a result, by adding the virtual user, the outer bound is attained.

Next, we consider the same scenario as in Fig. 11, but now with $N_{t}=3$ antennas per BS. After solving the optimization problem (37) and (41) for this setting, we can check that the DoF outer bound is $\frac{17}{6}$ DoF. The supersymbol of the nBIA scheme of Section IV is shown in Fig. 13. If we implement the beamforming matrices of Section IV, symbol extensions $\{31-40\}$ of user $p_{1,2}$ are idle. Therefore, as in the previous toy example, we can design new beamforming matrices that include transmission to a virtual user $v_{1,2}$ in order to get an additional DoF during each idle symbol extension. This way, the 3 private users achieve $3 \mathrm{DoF}$ in each of the 10 alignment blocks plus 10 additional DoF for virtual user $v_{1,2}$, which is the same physical user as $p_{1,2}$. Additionally, shared user $s h_{1}$ achieves 6 DoF in each of the 4 alignment blocks. In other words, the scheme attains $\frac{31}{11} \mathrm{DoF}$, which is only $\frac{1}{66}$ DoF below the outer bound. On the contrary, since cBIA does not leverage the partial connectivity of the system, it only achieves $\frac{5}{3}$ DoF.

For the general case, the construction of the supersymbol is the same as in an symmetric setting where $K_{p}=K_{p, n_{\max }}$ with $K_{p, n_{\max }}=\max _{n}\left\{K_{p, n}\right\}$. S-Block 1 consists of $L_{S-B 1}=$

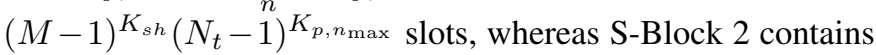
$L_{S-B 2}=t_{s h, \max }+t_{p, \max }$ symbol extensions where

$$
t_{p, \max }=K_{p, n_{\max }}\left[(M-1)^{K_{s h}}\left(N_{t}-1\right)^{K_{p, n_{\max }}-1}\right]
$$

and

$$
t_{s h, \max }=K_{s h}\left[(M-1)^{K_{s h}-1}\left(N_{t}-1\right)^{K_{p, n_{\max }}}\right] .
$$

Thus, there are

$$
\frac{t_{p, \max }}{K_{p, n_{\max }}} \sum_{n=1}^{N_{B S}} K_{p, n}
$$

alignment blocks during which the nBIA scheme of Section IV is applied. Moreover,

$$
\frac{t_{p, \max }}{K_{p, n_{\max }}} \sum_{n=1}^{N_{B S}}\left(K_{p, n_{\max }}-K_{p, n}\right)
$$

additional DoF are attained by taking advantage of the idle slots of S-Block 2. To do so, as in the toy examples, the beamforming matrices have to be modified in order to include transmission to virtual users. The sum DoF per symbol extension that are attained by the extension of the nBIA to the asymmetric setting are given by (47).

\section{ACHIEVABLE RATES}

So far, this work has focused on the high SNR regime and on the achievable DoF. To complement the previous sections, we derive closed-form expressions for the achievable rates of the nBIA scheme in the symmetric scenario for finite SNR. Expressions for the asymmetric scenario can be derived using the same procedure.

Due to the symmetry of the setting with respect to the private users, we analyze one alignment block of private user $p_{k, n}$. For simplicity, the index refers to the position of the symbol extension in the alignment block of $p_{k, n}$. First, recall that the supersymbol of $p_{k, n}$ has $t_{p} / K_{p}$ alignment blocks, each formed by $N_{t}$ symbol extensions. The first $N_{t}-1$ symbol extensions are contained in S-Block 1 and are subject to interference from the signals sent to $K_{p}-1$ private and $K_{s h}$ shared users. On the contrary, the last symbol extensions of the alignment blocks are in S-Block 2, are free of interference and are used to achieve decodability and measure the interference. Furthermore, since the beamforming matrix and the pattern of the channel modes are reused by the private users across the cells, the interference due to transmission to users $\left\{p_{j, n^{\prime}}\right\}_{n^{\prime}=1, n^{\prime} \neq n}^{N_{B S}}$ with $j \neq k$ is also removed together with the interference generated by the transmission to $p_{j, n}$. However, due to the reuse, the transmission to user $p_{k, n^{\prime}}$ generates a weak interference term that cannot be canceled, and is treated as noise. Hence, the received signal $\tilde{\mathbf{y}}^{\left[p_{k, n}\right]}=$

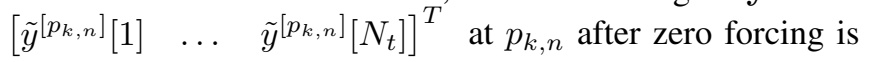

$\tilde{\mathbf{y}}_{\ell}^{\left[p_{k, n}\right]}=\mathbf{H}^{\left[p_{k, n}, n\right]} \mathbf{u}_{\ell}^{\left[p_{k, n}, n\right]}$

$$
+\sum_{n^{\prime}=1, n^{\prime} \neq n}^{N_{B S}} \sqrt{\alpha_{n^{\prime}}^{\left[p_{k, n}, n\right]}} \mathbf{H}^{\left[p_{k, n}, n^{\prime}\right]} \mathbf{u}_{\ell}^{\left[p_{k, n}, n^{\prime}\right]}+\tilde{\mathbf{z}}^{\left[p_{k, n}\right]},
$$




$$
\begin{aligned}
\operatorname{DoF}_{\mathrm{nBIA}, \text { asymm }}= & \frac{M \frac{t_{s h, \max }}{K_{s h}} K_{s h}+N_{t} \frac{t_{p, \max }}{K_{p, n_{\max }}} \sum_{n=1}^{N_{B S}} K_{p, n}+\frac{t_{p, \max }}{K_{p, n_{\max }}} \sum_{n=1}^{N_{B S}}\left(K_{p, n_{\max }}-K_{p, n}\right)}{L_{S-B 1}+L_{S-B 2}} \\
& =\frac{M K_{s h}\left(N_{t}-1\right)+(M-1)\left[\left(N_{t}-1\right) \sum_{n=1}^{N_{B S}} K_{p, n}+N_{B S} K_{p, n_{\max }}\right]}{(M-1)\left(N_{t}-1\right)+K_{s h}\left(N_{t}-1\right)+K_{p, n_{\max }}(M-1)} .
\end{aligned}
$$

where $\alpha_{n^{\prime}}^{\left[p_{k, n}, n\right]}$ is the relative power of the signal of BS $n^{\prime}$ received at user $p_{k, n}$ taking the power of the signal received from BS $n$ as reference, i.e. $\alpha_{n}^{\left[p_{k, n}, n\right]}=1$. In (48), $\mathbf{H}^{\left[p_{k, n}, n^{\prime}\right]}=$ $\left[\begin{array}{lll}\mathbf{h}^{\left[p_{k, n}, n^{\prime}\right]}(1)^{T} & \ldots & \mathbf{h}^{\left[p_{k, n}, n^{\prime}\right]}\left(N_{t}\right)^{T}\end{array}\right]^{T} \in \mathbb{C}^{N_{t} \times N_{t}}$ contains the channel coefficients between $p_{k, n}$ and BS $n^{\prime}$ normalized by $\sqrt{\alpha_{n^{\prime}}^{\left[p_{k, n}, n\right]}}$ and $\tilde{\mathbf{z}}^{\left[p_{k, n}\right]} \in \mathbb{C}^{N_{t} \times 1}$ is the noise vector after interference subtraction. Consequently, the $N_{t}$-th element of $\tilde{\mathbf{z}}^{\left[p_{k, n}\right]}$ is $\tilde{z}_{N_{t}}^{\left[p_{k, n}\right]}=z^{\left[p_{k, n}\right]}\left[N_{t}\right]$, while

$$
\tilde{z}_{i}^{\left[p_{k, n}\right]}=z^{\left[p_{k, n}\right]}[i]-\sum_{j=1 ; j \neq k}^{K_{p}} z^{\left[p_{j, n}\right]}[i]-\sum_{k^{\prime}=1}^{K_{s h}} z^{\left[s h_{k^{\prime}}\right]}[i]
$$

for any $i \in\left\{1,2, \ldots, N_{t}-1\right\}$. From now on, we will assume that the noise terms $z^{\left[p_{k, n}\right]}[i]$ and $z^{\left[s h_{k^{\prime}}\right]}[i]$ are independent and that $z^{\left[p_{k, n}\right]}[i], z^{\left[s h_{k^{\prime}}\right]}[i] \sim \mathcal{C N}(0,1)$ for all $p_{k, n} \in \mathcal{K}_{p, n}$, $s h_{k} \in \mathcal{K}_{s h}$ and $n \in\left\{1,2, \ldots, N_{B S}\right\}$.

Since the length of S-Block 1 is $\left(N_{t}-1\right)^{K_{p}}(M-1)^{K_{s h}}$ and each BS serves $K_{p}+K_{s h}$ users at each slot, $N_{t}\left(K_{p}+\right.$ $\left.K_{s h}\right)\left(N_{t}-1\right)^{K_{p}}(M-1)^{K_{s h}}$ symbols are sent over S-Block 1. On the other hand, to allow decodability and interference cancellation, S-Block 2 provides an additional symbol extension per alignment block in an orthogonal fashion. Since there are $t_{s h}$ and $t_{p}$ alignment blocks per $K_{p}$ private and per $K_{s h}$ shared users, respectively, to exploit the partial connectivity each BS needs to transmit $N_{t}\left(t_{p}+t_{s h}\right)$ symbols during S-Block 2 . Therefore, assuming equal power transmission to each stream, the allocated power per symbol is given by $(50) .^{2}$ Moreover, since each supersymbol contains $t_{p} / K_{p}$ alignment blocks per private user (see (23)), the ratio of alignment blocks per private user over the total number of slots is

$$
B_{p}=\frac{M-1}{(M-1)\left(N_{t}+K_{p}-1\right)+K_{s h}\left(N_{t}-1\right)} .
$$

Thus, the normalized rate per slot for $p_{k, n}$ is

$$
R^{\left[p_{k, n}\right]}=B_{p} \mathbb{E}\left[\log \operatorname{det}\left(\mathbf{I}+P_{s t r} \mathbf{A}^{\left[p_{k, n}, n\right]} \mathbf{R}_{\tilde{z}}^{\left[p_{k, n}\right]}\right)\right]
$$

where $\mathbf{A}^{\left[p_{k, n}, n\right]}=\mathbf{H}^{\left[p_{k, n}, n\right]} \mathbf{H}^{\left[p_{k, n}, n\right]}{ }^{H}$,

$$
\mathbf{R}_{\tilde{z}}^{\left[p_{k, n}\right]}=\mathbf{R}_{z}^{\left[p_{k, n}\right]}+P_{s t r} \sum_{n^{\prime}=1, n^{\prime} \neq n}^{N_{B S}} \alpha_{n^{\prime}}^{\left[p_{k, n}\right]} \mathbf{A}^{\left[p_{k, n}, n^{\prime}\right]},
$$

and $\mathbf{R}_{z}^{\left[p_{k, n}\right]}=\left[\begin{array}{cc}\left(K_{p}+K_{s h}\right) \mathbf{I}_{N_{t}-1} & \mathbf{0}_{N_{t}-1,1} \\ \mathbf{0}_{1, N_{t}-1} & 1\end{array}\right]$.

Similarly, to obtain the rate expression for the shared users, recall that each alignment block of $s h_{k^{\prime}}$ is made up of

\footnotetext{
${ }^{2}$ Another alternative is to use the scheme of [12] that assigns equal power in each slot.
}

$M$ symbol extensions. The first $M-1$ symbol extensions are subject to interference by the signals sent to $N_{B S} \cdot K_{p}$ private users and $K_{s h}-1$ shared users, whereas the last slot is free of interference. In this case, BIA codes are not reused among shared users, and therefore interference from transmission to the private and the remaining shared users can be canceled entirely. Thus, the signal $\tilde{\mathbf{y}}^{\left[s h_{k^{\prime}}\right]}=$ $\left[\begin{array}{lll}\tilde{y}^{\left[s h_{k^{\prime}}\right]}[1] & \ldots & \tilde{y}^{\left[s h_{k^{\prime}}\right]}[M]\end{array}\right]^{T}$ after zero forcing is

$$
\tilde{\mathbf{y}}_{\ell^{\prime}}^{\left[s h_{k^{\prime}}\right]}=\mathbf{H}^{\left[s h_{k^{\prime}}\right]} \mathbf{u}_{\ell^{\prime}}^{\left[s h_{k^{\prime}}\right]}+\tilde{\mathbf{z}}^{\left[s h_{k^{\prime}}\right]}
$$

where $\mathbf{H}^{\left[s h_{k^{\prime}}\right]}=\left[\begin{array}{lll}\mathbf{h}^{\left[s h_{k^{\prime}}\right]}(1)^{T} & \ldots & \mathbf{h}^{\left[s h_{k^{\prime}}\right]}(M)^{T}\end{array}\right]^{T} \in$ $\mathbb{C}^{M \times M}$ and

$$
\begin{aligned}
\mathbf{h}^{\left[s h_{k^{\prime}}\right]}(m)=\left[h_{1}^{\left[s h_{k^{\prime}}, 1\right]}(m)^{T}, \ldots, \sqrt{\beta_{n}^{\left[s h_{k^{\prime}}\right]}} h_{t}^{\left[s h_{k^{\prime}}, n\right]}(m)^{T}\right. \\
\left., \ldots, \sqrt{\beta_{N_{B S}}^{\left[s h_{k^{\prime}}\right]}} h_{N_{t}}^{\left[s h_{k^{\prime}}, N_{B S}\right]}(m)^{T}\right]^{T} \in \mathbb{C}^{M \times 1}
\end{aligned}
$$

contains the coefficients of the channel between user $s h_{k^{\prime}}$ and the $N_{t} N_{B S}$ antennas for mode $m, \beta_{m}^{\left[s h_{k^{\prime}}\right]}$ denotes the relative power of the signal of BS $m$ received at user $s h_{k^{\prime}}$ taking the power of the signal received from BS 1 as reference, i.e. $\beta_{1}^{\left[s h_{k^{\prime}}\right]}=1$, and $\tilde{\mathbf{z}}^{\left[s h_{k^{\prime}}\right]} \in \mathbb{C}^{M \times 1}$ is the noise vector after zero forcing, whose structure is similar to $\tilde{\mathbf{z}}^{\left[p_{k, n}\right]}$. Notice that the first $(M-1)$ terms are subject to a noise increment $K_{p}+$ $K_{s h}-1$ due to interference subtraction, while the $M$-th term only contains the noise term $z^{\left[s h_{k^{\prime}}\right]}\left[N_{t}\right]$.

The power allocated to each symbol is also given by (50). Moreover, $t_{s h} / K_{s h}$ alignment blocks are used to transmit to each shared user (see (20)). Hence, the ratio of alignment blocks per shared user over the supersymbol length is

$$
B_{s h}=\frac{N_{t}-1}{(M-1)\left(N_{t}+K_{p}-1\right)+K_{s h}\left(N_{t}-1\right)} .
$$

Therefore, the normalized rate of $s h_{k^{\prime}}$ is

$$
R^{\left[s h_{k^{\prime}}\right]}=B_{s h} \mathbb{E}\left[\log \operatorname{det}\left(\mathbf{I}+P_{s r t} \mathbf{A}^{\left[s h_{k^{\prime}}\right]} \mathbf{R}_{z}^{\left[s h_{k^{\prime}}\right]}-1\right)\right]
$$

where $\mathbf{A}^{\left[s h_{k^{\prime}}\right]}=\mathbf{H}^{\left[s h_{k^{\prime}}\right]} \mathbf{H}^{\left[s h_{k^{\prime}}\right]^{H}}$ and

$$
\mathbf{R}_{z}^{\left[s h_{k^{\prime}}\right]}=\left[\begin{array}{cc}
\left(K_{p}+K_{s h}\right) \mathbf{I}_{M-1} & \mathbf{0}_{M-1,1} \\
\mathbf{0}_{1, M-1} & 1
\end{array}\right] .
$$

\section{Simulation Results}

The achievable DoF for different transmission schemes over a symmetric partially connected network are depicted in Fig. 14. A two-cell scenario where each BS is equipped with $N_{t}=$ 3 antennas is assumed; there are 3 private users per each shared user in each cell, i.e. in the first iteration $K_{p, 1}=K_{p, 2}=$ 3 and $K_{s h}=2$. As expected, the proposed nBIA scheme 


$$
P_{s t r}=\frac{\left(N_{t}-1\right)(M-1)+K_{p}(M-1)+K_{s h}\left(N_{t}-1\right)}{N_{t}\left[\left(K_{p}+K_{s h}\right)\left(N_{t}-1\right)(M-1)+K_{p}(M-1)+K_{s h}\left(N_{t}-1\right)\right]} P .
$$

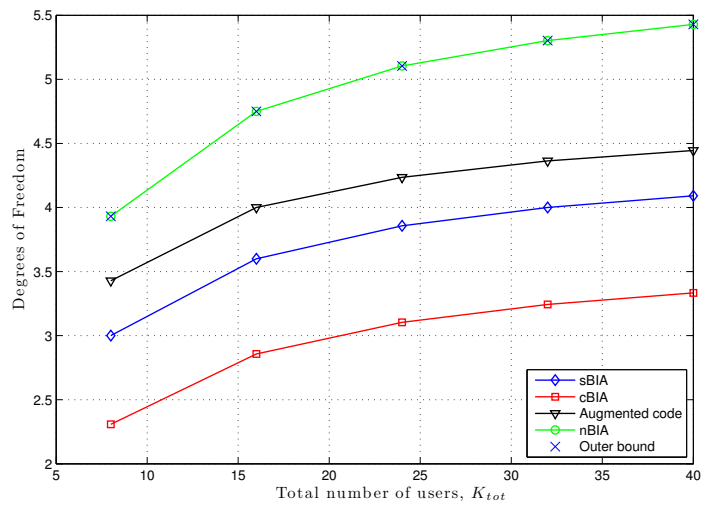

Fig. 14. Achievable DoF over a symmetric partially connected network. $N_{B S}=2, N_{t}=3$, and $K_{p, 1}=K_{p, 2}=\frac{3}{2} K_{s h}$.

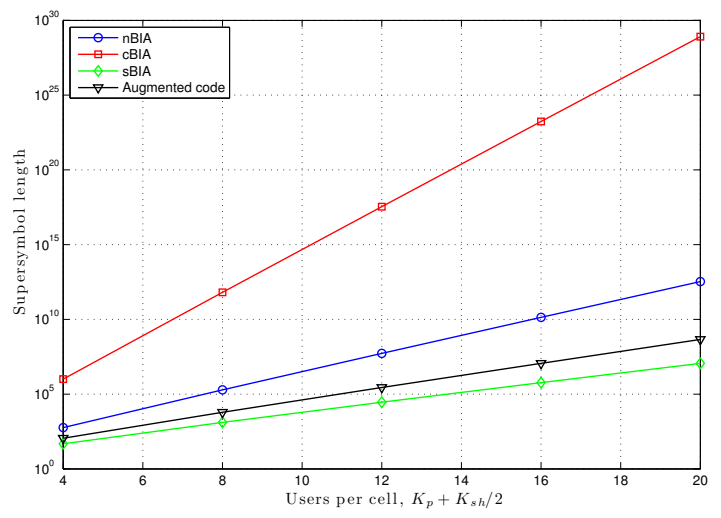

Fig. 15. Comparison of the supersymbol length. $N_{B S}=2, N_{t}=3$, and $K_{p, 1}=K_{p, 2}=\frac{3}{2} K_{s h}$.

achieves the information theoretic outer bound of Section V. In contrast, although the DoF grow with the number of users, the performance of cBIA is inferior due to the lack of connectivity. Besides, since in the augmented code solution proposed in [14] the shared users are not subject to intercell interference, the scheme attains more DoF compared to the case where sBIA is implemented in each cell. However, its performance is inferior to the proposed nBIA scheme.

The length of the supersymbols of the different transmission schemes is shown in Fig. 15 for the same parameters as in Fig. 14. As can be seen, the implementation of cBIA requires a prohibitive supersymbol length. On the other hand, the shortest supersymbol corresponds to an independent implementation of the sBIA scheme at each cell, which does not deal with the intercell interference. The proposed nBIA scheme has an acceptable supersymbol length with similar slope as sBIA and augmented code. This advantage is more remarkable taking into account the DoF achieved by nBIA in comparison with other schemes.

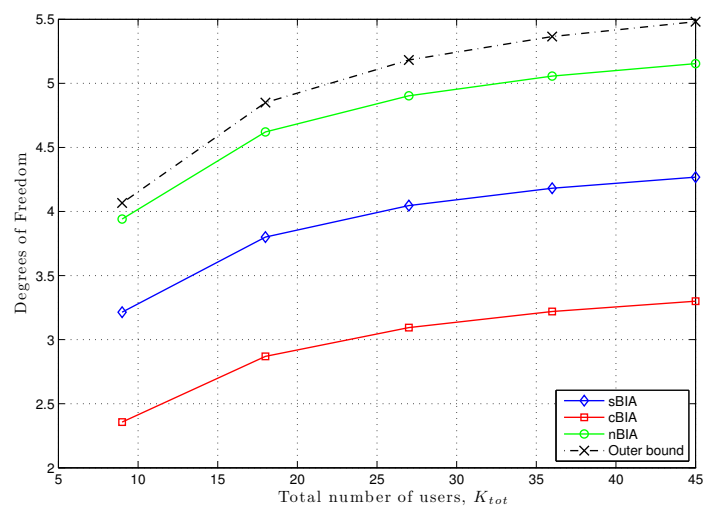

Fig. 16. Achievable DoF for an asymmetric partially connected network. $N_{B S}=2, N_{t}=3$, and $K_{p_{\max }}=\frac{4}{3} K_{p_{\min }}=2 K_{s h}$.

In Fig. 16 we show the achievable DoF for an asymmetric two-cell scenario where one cell contains $\frac{4}{3}$ times the private users of the other cell. As pointed out in Section VI, the nBIA scheme does not always achieve the outer bound of Section V. However it is close to the sum-DoF outer bound. Moreover, it can be seen that nBIA attains more DoF than other schemes. ${ }^{3}$ In contrast with nBIA, cBIA attains significantly fewer DoF since it does not exploit the lack of full connectivity.

The achievable sum rate of the users in each cell, i.e. $K_{\text {cell }}=K_{p}+\frac{K_{s h}}{2}$ in a two-cell scenario is plotted in Fig. 17. Each BS is equipped with $N_{t}=3$ antennas that serve a fixed number of private users, $K_{p}=6$. The transmission power is fixed at $25 \mathrm{~dB}$ and the average Signal-to-Interference Ratio (SIR) is assumed to be $10 \mathrm{~dB}$ and $2 \mathrm{~dB}$ for the private and the shared users, respectively. The nBIA scheme achieves a larger sum rate than the other schemes. Furthermore, the sum rate increases with the number of shared users. Notice that the cBIA scheme achieves a poor sum rate in comparison with the other schemes because many interference terms have to be subtracted. In comparison with augmented code and sBIA, it can be seen that nBIA has better performance, especially when the number of shared users, $K_{s h}$, is large.

\section{Conclusions}

In this work a novel Blind Interference Alignment scheme based on reconfigurable antennas is developed for cellular networks with partial connectivity. The proposed scheme allows removal of all intracell and intercell interference without any knowledge of the channel state information at the transmitter. For symmetric settings where the number of private users per cell is the same, this scheme achieves the information theoretic Degrees-of-Freedom bound, which is larger than the DoF achieved when employing a fully cooperative blind interference alignment scheme over a fully connected network.

\footnotetext{
${ }^{3}$ Since augmented code was devised for symmetric networks, its performance is not depicted.
} 


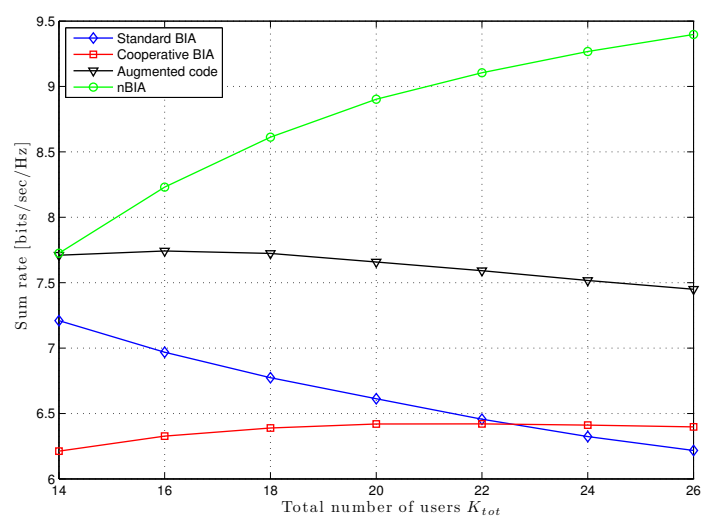

Fig. 17. Average achievable sum rates per cell versus the number of shared users $K_{s h}$. The SNR is fixed to $25 \mathrm{~dB}$ for all users, whereas the average SIR is $10 \mathrm{~dB}$ and $2 \mathrm{~dB}$ for private and shared users, respectively. $N_{B S}=2$, $N_{t}=3$, and $K_{p}=6$.

Moreover, it is also shown that the proposed scheme can be DoF optimal for some asymmetric settings. This improvement in performance is achieved by appropriately combining two blind interference alignment schemes corresponding to the private and the shared users in a way that leverages the partial connectivity. Furthermore, a considerable reduction of the supersymbol length is attained in comparison with the fully cooperative scheme, rendering the scheme more robust to temporal and frequency variations of the channel.

\section{REFERENCES}

[1] V. R. Cadambe and S. A. Jafar, "Interference alignment and the degrees of freedom for the $K$-user interference channel," IEEE Trans. Inf. Theory, vol. 54, no. 8, pp. 3425-3441, 2008.

[2] S. A. Jafar, "Blind interference alignment," IEEE Journ. Select. Topics Signal Processing, vol. 6, no. 3, pp. 216-227, 2012.

[3] S. A. Jafar and S. Shamai, "Degrees of freedom region of the MIMO X channel," IEEE Trans. Inf. Theory, vol. 54, no. 1, pp. 151-170, 2008.

[4] T. Gou and S. A. Jafar, "Degrees of freedom of the $K$ user $M \times N$ MIMO interference channel," IEEE Trans. Inf. Theory, vol. 55, no. 12, pp. 6040-6057, 2010.

[5] S. A. Jafar, Interference Alignment: A New Look at Signal Dimensions in a Communication Network. Now Publishers, 2011.

[6] S. A. Ramprashad, G. Caire, and H. Papadopoulos, "Cellular and network MIMO architectures MU-MIMO spectral efficiency and costs of channel state information," in IEEE Asilomar Conf. Sig., Sys., Computers, 2009, pp. 1811-1818.

[7] T. Gou, C. Wang, and S. A. Jafar, "Aiming perfectly in the darkblind interference alignment through staggered antenna switching," IEEE Trans. Signal Process., vol. 59, no. 6, pp. 2734-2744, 2011.

[8] — - "Interference alignment through staggered antenna switching for MIMO BC with no CSIT," in IEEE Asilomar Conf. Sig., Sys., Computers, 2010, pp. 2081-2085.

[9] T. Gou, S. A. Jafar, and C. Wang, "On the degrees of freedom of finite state compound wireless networks," IEEE Trans. Inf. Theory, vol. 57, no. 6, pp. 3286-3308, 2011.

[10] Q. Rongrong, M. Sellathurai, and D. Wilcox, "On the design of blind interference alignment using ESPAR antenna," in International ICST Conf. Commun. and Networking in China (CHINACOM), 2012, pp. 866870.

[11] C. Wang, H. C. Papadopoulos, S. A. Ramprashad, and G. Caire, "Design and operation of blind interference alignment in cellular and clusterbased systems," in Inf. Theory and Applications Workshop (ITA), 2011, pp. $1-10$.

[12] _ , "Improved blind interference alignment in a cellular environment using power allocation and cell-based clusters," in IEEE Int. Conf. Commun. (ICC), 2011, pp. 1-6.
[13] Q. Spencer, A. Swindlehurst, and M. Haardt, "Zero-forcing methods for downlink spatial multiplexing in multiuser MIMO channels," IEEE Trans. Signal Process., vol. 52, no. 2, pp. 461-471, 2004.

[14] S. Akoum, C. S. Chen, M. Debbah, and R. W. Heath Jr., "Data sharing coordination and blind interference alignment for cellular networks," in IEEE Global Commun. Conf. (GLOBECOM), 2012, pp. 4273-4277.

[15] S. A. Jafar, "Topological interference management through index coding," CoRR, vol. abs/1301.3106, 2013.

[16] C. Geng, N. Naderializadeh, A. S. Avestimehr, and S. A. Jafar, "On the optimality of treating interference as noise," CoRR, vol. abs/1305.4610, 2013.

[17] T. Gou and S. A. Jafar, "Optimal use of current and outdated channel state information: Degrees of freedom of the MISO BC with mixed CSIT," IEEE Commun. Lett., vol. 16, no. 7, pp. 1084-1087, 2012. 